\title{
A comparison theorem for cosmological lightcones
}

\author{
Mauro Carfora ${ }^{1,2}$ [D $\cdot$ Francesca Familiari ${ }^{1,2}$ \\ Received: 14 December 2020 / Revised: 18 March 2021 / Accepted: 1 April 2021 / \\ Published online: 19 April 2021 \\ (C) The Author(s) 2021
}

\begin{abstract}
Let $(M, g)$ denote a cosmological spacetime describing the evolution of a universe which is isotropic and homogeneous on large scales, but highly inhomogeneous on smaller scales. We consider two past lightcones, the first, $\mathcal{C}_{L}^{-}(p, g)$, is associated with the physical observer $p \in M$ who describes the actual physical spacetime geometry of $(M, g)$ at the length scale $L$, whereas the second, $\mathcal{C}_{L}^{-}(p, \hat{g})$, is associated with an idealized version of the observer $p$ who, notwithstanding the presence of local inhomogeneities at the given scale $L$, wish to model $(M, g)$ with a member $(M, \hat{g})$ of the family of Friedmann-Lemaitre-Robertson-Walker spacetimes. In such a framework, we discuss a number of mathematical results that allows a rigorous comparison between the two lightcones $\mathcal{C}_{L}^{-}(p, g)$ and $\mathcal{C}_{L}^{-}(p, \hat{g})$. In particular, we introduce a scaledependent $(L)$ lightcone-comparison functional, defined by a harmonic type energy, associated with a natural map between the physical $\mathcal{C}_{L}^{-}(p, g)$ and the FLRW reference lightcone $\mathcal{C}_{L}^{-}(p, \hat{g})$. This functional has a number of remarkable properties, in particular it vanishes iff, at the given length-scale, the corresponding lightcone surface sections (the celestial spheres) are isometric. We discuss in detail its variational analysis and prove the existence of a minimum that characterizes a natural scale-dependent distance functional between the two lightcones. We also indicate how it is possible to extend our results to the case when caustics develop on the physical past lightcone $\mathcal{C}_{L}^{-}(p, g)$. Finally, by exploiting causal diamond theory, we show how the distance functional is related (to leading order in the scale $L$ ) to spacetime scalar curvature in the causal past of the two lightcones, and briefly illustrate a number of its possible applications.
\end{abstract}

Keywords Lightcone cosmography $\cdot$ Mathematical cosmology $\cdot$ Cosmological backreaction · Lightcones

$凶 \quad$ Mauro Carfora

mauro.carfora@unipv.it

Francesca Familiari

francesca.familiari01@universitadipavia.it

1 Department of Physics, University of Pavia, Pavia, Italy

2 (GNFM and INFN) Italian National Group of Mathematical Physics, and INFN Pavia Section, Pavia, Italy 
Mathematics Subject Classification 83 (Relativity and gravitational theory), 83F05 (Cosmology), 58Z05 (Applications of global analysis to the sciences)

\section{Introduction}

For many among us, the first encounter with Boris Dubrovin has been through his classic books Géometrie Contemporaine: méthodes et applications, coauthored with S. Novikov and A. Fomenko, and published by MIR in a silk-bonded three-volume set, an edition that, notwithstanding the later expanded Springer version [9], we treasured with care. To the best of our knowledge, Boris did not work in general relativity but in his Géometrie Contemporaine there are two wonderful little chapters, just short of a total of fifty tersely written pages, which provide the fastest way to acquaint yourself with general relativity. Thinking of these elegant pages, we hope that it is not inappropriate to dedicate to Boris the present work addressing a long-standing problem in mathematical cosmology.

Let us recall that the observed universe is described by a spacetime $(M, g)$, a fourdimensional manifold $M$ endowed with a Lorentzian metric $g$, which is (statistically) isotropic and homogeneous only on sufficiently large scales, say $L \geq L_{0}$, where the current acceptable figure for the homogeneity scale is $L_{0} \geq 100 \mathrm{~h}^{-1} \mathrm{Mpc}$, where $h$ is the dimensionless Hubble parameter describing the relative uncertainty of the true value of the present-epoch Hubble-Lemaitre constant $H_{0}=100 \mathrm{~h} \mathrm{Km} / \mathrm{s} / \mathrm{Mpc}$. At these homogeneity scales $(M, g)$ is described with great accuracy by a member of the homogeneous and isotropic family of Friedman-Lemaitre-Robertson-Walker (FLRW) spacetimes $(M, \hat{g})$. At smaller scales, where inhomogeneities statistically dominate, we should resort to the full-fledged spacetime geometry of $(M, g)$ in order to provide the correct dynamical description of cosmological observations. However, coming to mathematical terms with the geometrical and physical structure of $(M, g)$ is a daunting task and typically we keep on modeling the dynamics of the universe over these inhomogeneity scales with the FLRW model $(M, \hat{g})$, thought of as providing a background around which the actual spacetime geometry $(M, g)$ is perturbatively expanded. If we want to go beyond perturbation theory, we face the mathematically delicate problem of finding a way for comparing the past lightcone region ${ }^{1} \mathcal{C}_{L}^{-}(p, g)$, associated with an (instantaneous) observer $p$ sampling the inhomogeneities in $(M, g)$ at the given length scale $L$, with the corresponding past lightcone region $\mathcal{C}_{L}^{-}(p, \hat{g})$ in the assumed FLRW background $(M, \hat{g})$. In modern high-precision cosmology this is one of the most delicate issues when modeling the observed universe. Inspired by the foundational works by Ellis et al. [10,11], in this paper we provide a number of mathematical results that allow to compare the past lightcone regions $\mathcal{C}_{L}^{-}(p, g)$ and $\mathcal{C}_{L}^{-}(p, \hat{g})$. In particular, we introduce a scale-dependent conformal diffeomorphism $\varphi_{L}$ and a corresponding lightcone comparison functional $E_{\widehat{\Sigma} \Sigma}\left[\varphi_{L}\right]$ between the physical and the FLRW reference celestial spheres $\Sigma_{L} \subset \mathcal{C}_{L}^{-}(p, g)$ and $\widehat{\Sigma}_{L} \subset \mathcal{C}_{L}^{-}(p, \hat{g})$ probed, at the given length scale $L$, on the respective lightcones regions $\mathcal{C}_{L}^{-}(p, g)$ and

\footnotetext{
${ }^{1}$ Details on the notation adopted are explained in full detail in the body of the paper. In particular, the regions $\mathcal{C}_{L}^{-}(p, g)$ and $\mathcal{C}_{L}^{-}(p, \hat{g})$ are defined by (19).
} 
$\mathcal{C}_{L}^{-}(p, \hat{g})$. It is important to stress that the scale-dependent map $\varphi_{L}$, is not an abstract map, but it actually relates the physical observations on $\Sigma_{L}$ with those, described with a FLRW bias, on $\widehat{\Sigma}_{L}$. The functional $E_{\widehat{\Sigma} \Sigma}\left[\varphi_{L}\right]$ is defined by a harmonic map type energy and has a number of remarkable properties. In particular, it vanishes iff, at the given length-scale $L$, the corresponding lightcone surface sections $\Sigma_{L}$ and $\widehat{\Sigma}_{L}$ (which are topologically 2 -spheres, as long as null-caustics are absent) are isometric. Moreover, by taking the inf of $E_{\widehat{\Sigma} \Sigma}\left[\varphi_{L}\right]$ over a suitable class of extended maps $\varphi_{L}$ (extension necessary in order to account also for the presence of lightcone caustics), we provide a scale-dependent distance functional, $d_{L}[\widehat{\Sigma}, \Sigma]$, between the physical and the FLRW reference lightcones $\mathcal{C}_{L}^{-}(p, g)$ and $\mathcal{C}_{L}^{-}(p, \hat{g})$. This distance significantly extends the lightcone theorem proved in [7]. In order to characterize the physical interpretation of $E_{\widehat{\Sigma} \Sigma}\left[\varphi_{L}\right]$ we exploit small causal diamond theory, as described in [13], to show that in the caustic-free region near the tip $p$ of $\mathcal{C}_{L}^{-}(p, g)$ and $\mathcal{C}_{L}^{-}(p, \hat{g})$, namely for $L$ small enough, $d_{L}[\widehat{\Sigma}, \Sigma]$ is perturbatively related (at first order in the scale $L$ ), to the gravitational lensing distortion on $\mathcal{C}_{L}^{-}(p, g)$ and to the spacetime scalar curvatures $R(g)$ and $R(\hat{g})$ in the interior of these lightcones. This connection between $d_{L}[\widehat{\Sigma}, \Sigma]$ and the rich literature on causal diamond is a property that may play an important role in cosmological modeling, and the characterization of a full non-perturbative relation between the analysis presented in this paper and causal diamond theory is a relevant open problem to address ${ }^{2}$. In particular, it would be interesting to explore if there is a link between the functionals $E_{\widehat{\Sigma} \Sigma}\left[\varphi_{L}\right]$ and $d_{L}[\widehat{\Sigma}, \Sigma]$ introduced here and the comparison theorems for causal diamonds discussed in detail in the remarkable paper by Berthiere et al. [2]. Even if there is no direct relation between their approach and our motivation, geometric analysis techniques and results, the perturbative characterization mentioned above indicates that these two approaches may profitably interact. We believe more progress can be made in cosmological modeling if we join these two points of view. Finally, it must be stressed that the results presented here can be easily extended to fiducial reference metrics more general than those described by the FLRW family, e.g., to a homogeneous solution of Einstein equations. Our choice of a reference FLRW is naturally related to the actual prevalence of this family of metrics in discussing cosmological dynamics.

\section{Cosmological observers and observational coordinates along the past lightcones}

Throughout this paper $(M, g)$ denotes a cosmological spacetime where $g$ is a Lorentzian metric, and where $M$ is a smooth four-dimensional manifold which for our purposes we can assume diffeomorphic to $\mathbb{R}^{4}$. In local coordinates $\left\{x^{i}\right\}_{i=1}^{4}$, we write $g=g_{i k} \mathrm{~d} x^{i} \otimes \mathrm{d} x^{k}$, where the metric components $g_{i k}:=g\left(\partial_{i}, \partial_{k}\right)$ in the coordinate basis $\left\{\partial_{i}:=\partial / \partial x^{i}\right\}_{i=1}^{4}$, have the Lorentzian signature $(+,+,+,-)$, and the Einstein summation convention is in effect. We denote by $\nabla_{(g)}$ (or $\nabla$ if there is no danger of confusion) the Levi-Civita connection of $g$, and let $\mathcal{R} m(g)=\mathcal{R}_{k l m}^{i} \partial_{i} \otimes \mathrm{d} x^{k} \otimes \mathrm{d} x^{l} \otimes \mathrm{d} x^{m}$,

\footnotetext{
2 Preliminary results in this direction have been presented, as an invited talk, by one of us (MC) at the joint symposium "Cosmological model-building" during the DPG-Früjahrstagung 2019 München.
} 
$\mathcal{R} i c(g)=\mathcal{R}_{a b} \mathrm{~d} x^{a} \otimes \mathrm{d} x^{b}$ and $\mathcal{R}(g)$ be the corresponding Riemann, Ricci and scalar curvature operators, respectively. We assume that $(M, g)$ is associated with the evolution of a universe which is (statistically) isotropic and homogeneous on sufficiently large scales, whereas local inhomogeneities dominate over smaller scales. The massenergy content in $(M, g)$ is phenomenologically described by an energy-momentum tensor $T$ the explicit expression of which is not needed in our analysis, we only assume that its matter components characterize a Hubble flow that generates a family of preferred worldlines parametrized by proper time $\tau$

$$
\begin{aligned}
\gamma_{s}: \mathbb{R}_{>0} & \longrightarrow(M, g) \\
\tau & \longmapsto \gamma_{s}(\tau),
\end{aligned}
$$

and labeled by suitable comoving (Lagrangian) coordinates $s$. We set $c=1$, and denote by $\dot{\gamma}_{s}:=\frac{\mathrm{d} \gamma_{s}(\tau)}{\mathrm{d} \tau}$, with $g\left(\dot{\gamma}_{s}, \dot{\gamma}_{s}\right)=-1$, the corresponding 4-velocity field. For simplicity, we assume that the worldlines (1) are geodesics, i.e., $\nabla_{\dot{\gamma}_{s}} \dot{\gamma}_{s}=0$. This is the spacetime within which we can frame the actual cosmological data gathered from our past lightcone observations. If we adopt the weak form of the cosmological principle, $\left(M, g, \gamma_{s}\right)$ can be identified with the phenomenological background spacetime or phenomenological background solution $(P B S)$, according to the notation introduced in [21]. In the same vein, we define phenomenological observers the collection of observers $\left\{\gamma_{s}\right\}$ comoving with the Hubble flow.

\subsection{The phenomenological lightcone metric}

Since in our analysis we fix our attention on a given observer, we drop the subscript $s$ in (1)), and describe a finite portion of the observer's worldline with the timelike geodesic segment $\tau \longmapsto \gamma(\tau),-\delta<\tau<\delta$, for some $\delta>0$, where $p:=\gamma(\tau=0)$ is the selected observational event. To set up the appropriate coordinates along $\gamma(\tau)$, let $\left(T_{p} M, g_{p},\left\{E_{(i)}\right\}\right)$ be the tangent space to $M$ at $p$ endowed with a $g$-orthonormal frame $\left\{E_{(i)}\right\}_{i=1, \ldots, 4}, g_{p}\left(E_{(i)}, E_{(k)}\right)=\eta_{i k}$, where $\eta_{i k}$ is the Minkowski metric, and where $E_{(4)}:=\left.\dot{\gamma}(\tau)\right|_{\tau=0}$. Notice that by parallel transport, this basis can be propagated along $\gamma(\tau)$. Let us introduce the set of past-directed null vectors and the set of pastdirected causal vectors in $\left(T_{p} M, g_{p}\right)$ according to

$$
\begin{aligned}
& C^{-}\left(T_{p} M, g_{p}\right):=\left\{X=\mathbb{X}^{i} E_{(i)} \neq 0 \in T_{p} M \mid \mathbb{X}^{4}+r=0\right\}, \\
& \overline{C^{-}}\left(T_{p} M, g_{p}\right):=\left\{X=\mathbb{X}^{i} E_{(i)} \neq 0 \in T_{p} M \mid \mathbb{X}^{4}+r \leq 0\right\},
\end{aligned}
$$

where $r:=\left(\sum_{a=1}^{3}\left(\mathbb{X}^{a}\right)^{2}\right)^{1 / 2}$. We use these sets of vectors in order to introduce observational coordinates in (a region of) the causal past of $p, J^{-}(p, g)$, by exploiting the exponential mapping based at $p$,

$$
\exp _{p}: \overline{C^{-}}\left(T_{p} M, g_{p}\right) \longrightarrow \quad M
$$




$$
X \quad \longmapsto \quad \exp _{p}(X):=\lambda_{X}(1)
$$

where $\lambda_{X}:[0, \infty) \longrightarrow(M, g)$ is the past-directed causal geodesic emanating from the point $p$ with initial tangent vector $\dot{\gamma}_{X}(0)=X \in \overline{C^{-}}\left(T_{p} M, g_{p}\right)$. If we assume that the metric is sufficiently regular ${ }^{3}$, then there is a neighborhood $N_{0}(g)$ of 0 in $T_{p} M$ and a geodesically convex neighborhood of $p, U_{p} \subset(M, g)$, defined by all points $q \in M$ which are within the domain of injectivity of $\exp _{p}$, where we can introduce geodesic normal coordinates $\left(X^{i}\right)$ according to

$$
\begin{aligned}
X^{i}:=\mathbb{X}^{i} \circ \exp _{p}^{-1}: M \cap U_{p} & \longrightarrow \mathbb{R}^{4} \\
q & \longmapsto X^{i}(q):=\mathbb{X}^{i}\left(\exp _{p}^{-1}(q)\right)
\end{aligned}
$$

and where $\mathbb{X}^{i}\left(\exp _{p}^{-1}(q)\right)$ are the components, in the $g$-orthonormal frame $\left\{E_{(i)}\right\}$, of the vector $\exp _{p}^{-1}(q) \in T_{p} M$. In particular, if we consider the past lightcone $\mathcal{C}^{-}(p, g)$ with vertex at $p$, then away from the past null cut locus of $p$, i.e., away from the set of lightcone caustics, normal coordinates can be used to parametrize the past light cone region $\mathcal{C}^{-}(p, g) \cap U_{p}$,

$$
\begin{aligned}
\exp _{p}: C^{-}\left(T_{p} M, g_{p}\right) \cap N_{0}(g) & \longrightarrow \mathcal{C}^{-}(p, g) \cap U_{p} \\
X=\mathbb{X}^{i} E_{(i)} & \longmapsto \exp _{p}\left(\mathbb{X}^{i} E_{(i)}\right)=q \Rightarrow\left\{X^{i}(q)\right\}
\end{aligned}
$$

Similarly, by restricting $\exp _{p}$ to $\overline{C^{-}}\left(T_{p} M, g_{p}\right) \cap N_{0}(g)$ we can parametrize with normal coordinates the region $J^{-}(p, g) \cap U_{p}$ within the causal past $J^{-}(p, g)$ of $p$. In particular, we can foliate $J^{-}(p, g) \cap U_{p}$ with the family of past lightcones $\mathcal{C}^{-}(\gamma(\tau), g)$ associated with the events $\gamma(\tau) \cap U_{p},-\delta<\tau \leq 0$, along the observer past-directed worldline. We can specialize the normal coordinates so introduced by setting

$$
x^{1}:=r:=\sqrt{\sum_{a=1}^{3}\left(X^{a}\right)^{2}}, \quad x^{2}:=\theta\left(X^{a} / r\right), \quad x^{3}:=\varphi\left(X^{a} / r\right), \quad x^{4}:=\tau=X^{4}+r,
$$

where $\theta\left(X^{a} / r\right), \varphi\left(X^{a} / r\right), a=1,2,3$, denote the standard angular coordinates of the direction $\left(X^{a} / r\right)$ on the unit 2-sphere $\mathbb{S}^{2}$ in $T_{p} M$ and where, according to (2), $x^{4}=0$ corresponds to the light cone region $\mathcal{C}^{-}(p, g) \cap U_{p}$. Notice that at the vertex $p=\gamma(\tau=0)$, the coordinate function $x^{4}$ is not differentiable (but it is continuous).

Remark 1 Under the stated hypotheses, and as long as we stay away from the vertex $p$ and from its null cut locus, we have that the past lightcone region $\mathcal{C}^{-}(p, g) \cap U_{p} \backslash\{p\}$ is topologically foliated by the $r$-dependent family of two-dimensional surfaces $\Sigma(p, r)$, the celestial spheres at scale $r$, reached by the set of past directed null geodesics as

\footnotetext{
3 A Lipschitz condition for the metric components suffices.
} 
the affine parameter $r$ varies, i.e.,

$$
\Sigma(p, r):=\left\{\exp _{p}(r \underline{n}) \mid \underline{n}:=(\theta, \varphi) \in \mathbb{S}^{2} \subset T_{p} M\right\}
$$

Each $\Sigma(p, r)$ is topologically a 2-sphere endowed with the $r$-dependent family of two-dimensional Riemannian metrics

$$
h(r):=\left.\left(\left.\exp _{p}^{*} g\right|_{\Sigma(p, r)}\right)_{\alpha \beta} \mathrm{d} x^{\alpha} \mathrm{d} x^{\beta}\right|_{r}
$$

obtained by using the exponential map to pull back to $\mathbb{S}^{2} \subset T_{p} M$ the two-dimensional metric $\left.g\right|_{\Sigma(p, r)}$ induced on $\Sigma(p, r)$ by the embedding $\Sigma(p, r) \hookrightarrow(M, g)$. We normalize this metric by imposing that the angular variables $x^{\alpha}=(\theta, \varphi)$, in the limit $r \searrow 0$, reduce to the standard spherical coordinates on the unit 2 -sphere $\mathbb{S}^{2}$, i.e.,

$$
\left.\lim _{r \searrow 0}\right|_{x^{4}=0} \frac{h_{\alpha \beta}(r) \mathrm{d} x^{\alpha} \mathrm{d} x^{\beta}}{r^{2}}=\mathrm{d} \Omega^{2}:=\mathrm{d} \theta^{2}+\sin ^{2} \theta \mathrm{d} \varphi^{2} .
$$

For a physical interpretation [11], it is convenient to parametrize $h(r)$ as a sky-mapping metric

$$
h(r)=D^{2}(r)\left(\mathrm{d} \Omega^{2}+\mathcal{L}_{\alpha \beta}(r) \mathrm{d} x^{\alpha} \mathrm{d} x^{\beta}\right),
$$

where $\mathrm{d} \Omega^{2}$ is the unit radius round metric on $\mathbb{S}^{2}$ (see (11)), and the coordinates $\left\{x^{\alpha}\right\}_{\alpha=2,3}$ provide the direction of observation (as seen at $p$ ) of the astrophysical sources on the celestial sphere $\Sigma(p, r)$. The function $D(r)$ is the observer area distance defined by the relation $\mathrm{d} \mu_{h(r)}=D^{2}(r) \mathrm{d} \mu_{\mathbb{S}^{2}}$ where $\mathrm{d} \mu_{h(r)}$ is the pulled-back (via $\left.\exp _{p}\right)$ area measure of $\left(\Sigma(p, r),\left.g\right|_{\Sigma(p, r)}\right)$, (roughly speaking, $\mathrm{d} \mu_{h(r)}$ can be interpreted [11] as the cross-sectional area element at the source location as seen by the observer at $p$ ) and $\mathrm{d} \mu_{\mathbb{S}^{2}}$ is the area element on the unit round sphere $\mathbb{S}^{2} \in T_{p} M$ (i.e., the element of solid angle subtended by the source at the observer location $p$ ). In the same vein, the symmetric tensor field $\mathcal{L}_{\alpha \beta}(r)$, describing the distortion of the normalized metric $h(r) / D^{2}(r)$ with respect to the round metric $\mathrm{d} \Omega^{2}$, can be interpreted as the image distortion of the sources on $(\Sigma(p, r), h(r))$ as seen by the observer at $p$. This term, which in general is not trace-free, involves both the gravitational lensing shear [11] and the gravitational focusing of the light rays generating the local source image magnification. By taking into account these remarks, we have the following characterization of the past lightcone metric in a neighborhood of the point $p$.

Lemma 2 In the geometrical coordinates introduced above, the null geodesics generators of $\mathcal{C}^{-}(p, g) \cap U_{p}$ have equation $x^{4}=0, x^{\alpha}=$ const., and their tangent vector is provided by $\frac{\partial}{\partial x^{1}}$, with $\left(\exp ^{*} g\right)\left(\frac{\partial}{\partial x^{1}}, \frac{\partial}{\partial x^{1}}\right)=0$. Since $\frac{\partial}{\partial x^{1}}$ is past-directed we can introduce the normalization

$$
\lim _{r \searrow 0}\left(\exp ^{*} g\right)\left(\frac{\partial}{\partial x^{1}}, \dot{\gamma}\right)=1
$$


and write the restriction of the spacetime metric $g$ on $\mathcal{C}^{-}(p, g) \cap U_{p}$ according to ${ }^{4}$

$$
\left.g\right|_{x^{4}=0}=g_{44}\left(d x^{4}\right)^{2}+2 g_{14} d x^{1} d x^{4}+2 g_{4 \alpha} d x^{4} d x^{\alpha}+h_{\alpha \beta} d x^{\alpha} d x^{\beta},
$$

where $\alpha, \beta=2,3$, and where the components $g_{i k}\left(x^{i}\right):=\left(\exp ^{*} g\right)\left(\frac{\partial}{\partial x^{i}}, \frac{\partial}{\partial x^{k}}\right)$, and $h_{\alpha \beta}\left(x^{i}\right)$ are all evaluated for $x^{4}=0$.

As already stressed, the coordinates $\left\{x^{i}\right\}$ are singular at the vertex $\gamma(\tau=0)=p$ of the cone. A detailed analysis of the limit $r \searrow 0$, besides the standard assumptions we already made, is carried out in detail in the foundational paper [10] (see paragraph 3 ) and in [8], (see paragraphs 4.2.1-4.2.3-4.5, the results presented there are stated for the future lightcone, but they can be easily adapted to the past lightcone).

Remark 3 Clearly the lightcone metric (14) does not hold when caustics form; however, our final result involving the characterization of a distance functional between lightcones naturally extends to the case when caustics are present.

\subsection{The reference FLRW lightcone metric}

Along the physical metric $g$, we also introduce in $M$ the FLRW metric $\hat{g}$ and the family of global Friedmannian observers $\hat{\gamma}_{s}$ that, at the homogeneity scale, we can associate with the cosmological data. This is the global background solution $(G B S)$ according to [21]. In full generality the geodesics $\tau \longmapsto \gamma(\tau)$, and $\hat{\tau} \longmapsto \hat{\gamma}(\hat{\tau}),-\delta<, \tau, \hat{\tau}<\delta$, associated with the corresponding Hubble flow in $(M, g, \gamma)$ and $(M, \hat{g}, \hat{\gamma})$, will be distinct but, in line with the set up adopted here, we assume that they share a common observational event $p$. We normalize the proper times $\tau$ and $\hat{\tau}$ along $\gamma(\tau)$ and $\hat{\gamma}(\hat{\tau})$ so that at $\tau=0=\hat{\tau}$ we have $\gamma(0)=p=\hat{\gamma}(0)$. Hence, together with the coordinates $\left\{x^{i}\right\}$ in $\left(M, g, \gamma_{s}\right)$, describing the observational metric (14) on the past lightcone $\mathcal{C}^{-}(p, g) \cap U_{p}$, we introduce corresponding (normal) coordinates $\left\{Y^{k}\right\}$ in the reference $(M, \hat{g}, \hat{\gamma})$. With an obvious adaptation of the analysis for $(M, g)$, carried out in previous subsection, let $N_{0}(\hat{g})$ denote the domain of injectivity of the exponential mapping $\widehat{\exp }_{p}: T_{p} M \longrightarrow(M, \hat{g})$ based at the event $p=\hat{\gamma}(0)$. If $\hat{U}_{p} \subset(M, \hat{g})$ denotes the region of injectivity of $\widehat{\exp }_{p}$ we can consider normal coordinates

$$
Y^{i}:=\mathbb{Y}^{i} \circ \widehat{\exp }_{p}^{-1}:(M, \hat{g}) \cap \hat{U}_{p} \longrightarrow \mathbb{R}
$$

where $\mathbb{Y}^{i}$ are the components of the vectors $\mathbb{Y} \in T_{p} M$ with respect to a $\hat{g}$ orthonormal frame $\left\{\hat{E}_{(i)}\right\}_{i=1, \ldots, 4}$ with $\hat{E}_{(4)}:=\hat{\dot{\gamma}}(0)$. Within $\hat{U}_{p}$ we can introduce, in full analogy with (7) and (14), the coordinates $y^{1}:=\hat{r}=\left(\sum_{a=1}^{3}\left(Y^{a}\right)^{2}\right)^{1 / 2}$, $\left.y^{\alpha}\right|_{\alpha=2,3}=\left(\theta\left(Y^{a} / \hat{r}\right), \varphi\left(Y^{a} / \hat{r}\right)\right)$ and parametrize $\mathcal{C}^{-}(p, \hat{g}) \cap \hat{U}_{p}$ in terms of the two-dimensional spheres

$$
\hat{\Sigma}(p, \hat{r}):=\left\{\exp _{p}(\hat{r} \underline{n}) \mid \underline{n}:=(\theta, \varphi) \in \mathbb{S}^{2} \subset T_{p} M\right\},
$$

\footnotetext{
4 A detailed and very informative analysis of geodesic coordinates along the past light cone is provided by [12].
} 
endowed with the round metric

$$
\hat{h}(\hat{r}):=\left.(\hat{g})_{\alpha \beta} \mathrm{d} y^{\alpha} \mathrm{d} y^{\beta}\right|_{\hat{r}}=a^{2}(\hat{r}) \hat{r}^{2}\left(\mathrm{~d} \theta^{2}+\sin ^{2} \theta \mathrm{d} \varphi^{2}\right),
$$

where $a(\hat{r})$ is the FLRW expansion factor corresponding to the distance $\hat{r}$. Hence, we can write the metric $\hat{g}$ on the reference FLRW past lightcone region $\mathcal{C}^{-}(p, \hat{g}) \cap \hat{U}_{p}$ as

$$
\left.\hat{g}\right|_{y^{4}=0}=\hat{g}_{44}\left(\mathrm{~d} y^{4}\right)^{2}+\hat{h}_{\alpha \beta} \mathrm{d} y^{\alpha} \mathrm{d} y^{\beta} \text {. }
$$

\section{Comparing lightcones: a scale dependent comparison functional}

According to our hypotheses, the spacetime $\left(M, g, \gamma_{s}\right)$ describes the evolution of a universe which is isotropic and homogeneous only at sufficiently large scales $L_{0}$. At these homogeneity scales, $\left(M, g, \gamma_{s}\right)$ is modeled by the FLRW spacetime $\left(M, \hat{g}, \hat{\gamma}_{s}\right)$. Even if at smaller scales, where inhomogeneities statistically dominate, $\left(M, g, \gamma_{s}\right)$ provides the bona fide spacetime describing cosmological observations, we can still use the reference $\left(M, \hat{g}, \hat{\gamma}_{s}\right)$ as a background FLRW model. As the observational length scale $L$ varies from the local highly inhomogeneous regions to the homogeneity scale $L_{0}$, we do not assume a priori that $\left(M, g, \gamma_{s}\right)$ is perturbatively near to the reference FLRW spacetime $\left(M, \hat{g}, \hat{\gamma}_{s}\right)$. Rather, we compare $\left(M, g, \gamma_{s}\right)$ with $\left(M, \hat{g}, \hat{\gamma}_{s}\right)$ keeping track of the pointwise and global relations among the various geometric quantities involved. In particular, we will compare the lightcone region $\mathcal{C}^{-}(p, g) \cap U_{p}$ with the reference FLRW lightcone region $\mathcal{C}^{-}(p, \hat{g}) \cap \hat{U}_{p}$, assuming that in such a range there are no lightcone caustics. As already emphasized, this is an assumption that makes easier to illustrate some of the technical arguments presented here, in the final part of the paper we indicate how our main result, concerning the existence and the properties of the distance functional described in the introduction, holds also in the more general case when caustics are present. That said, let us consider the following scale-dependent subsets of the past light cones $\mathcal{C}^{-}(p, g)$ and $\mathcal{C}^{-}(p, \hat{g})$,

$$
\mathcal{C}_{L}^{-}(p, g):=\exp _{p}\left[C_{L \leq L_{0}}^{-}\left(T_{p} M, g_{p}\right)\right], \mathcal{C}_{L}^{-}(p, \hat{g}):=\widehat{\exp }_{p}\left[C_{L \leq L_{0}}^{-}\left(T_{p} M, \hat{g}_{p}\right)\right],
$$

where

$$
\begin{aligned}
& C_{L \leq L_{0}}^{-}\left(T_{p} M, g_{p}\right):=\left\{X=\mathbb{X}^{i} E_{(i)} \in\left(T_{p} M, g_{p}\right) \mid X^{4}+r=0,-L_{0} \leq X^{4} \leq 0\right\}, \\
& C_{L \leq L_{0}}^{-}\left(T_{p} M, \hat{g}_{p}\right):=\left\{Y=\mathbb{Y}^{a} \hat{E}_{(a)} \in\left(T_{p} M, \hat{g}_{p}\right) \mid Y^{4}+\hat{r}=0,-L_{0} \leq Y^{4} \leq 0\right\},
\end{aligned}
$$

are the exponential map domains associated with the observational length-scale $L$ up to the homogeneity scale $L_{0}$. Under the stated caustic-free assumption, both $\mathcal{C}_{L}^{-}(p, g)$ and $\mathcal{C}_{L}^{-}(p, \hat{g})$ can be foliated in terms of the two-dimensional surfaces $\Sigma(p, r)$ and 
$\hat{\Sigma}(p, \hat{r})$ introduced in the previous section, i.e., we can write

$$
\mathcal{C}_{L}^{-}(p, g)=\bigcup_{0 \leq r \leq L_{0}} \Sigma(p, r), \quad \mathcal{C}_{L}^{-}(p, \hat{g})=\bigcup_{0 \leq \hat{r} \leq L_{0}} \hat{\Sigma}(p, \hat{r}) .
$$

On $\mathcal{C}_{L}^{-}(p, g)$ and $\mathcal{C}_{L}^{-}(p, \hat{g})$ the normal coordinates ${ }^{5}\left\{x^{i}\right\}$ and $\left\{y^{a}\right\}$, associated with the observational metric (14) and the reference metric (18), cannot be directly identified since they are defined in terms of the distinct exponential mappings $\exp _{p}$ and $\widehat{\exp }_{p}$ and, for a given initial tangent vector $X \in C_{L \leq L_{0}}^{-}\left(T_{p} M, g_{p}\right) \cap C_{L \leq L_{0}}^{-}\left(T_{p} M, \hat{g}_{p}\right)$, we have

$$
\exp _{p}(X)=q \neq \widehat{\exp }_{p}(X)=\hat{q} .
$$

However, $q$ and $\hat{q}$ are in the open spacetime region defined by

$$
M_{p}:=\exp _{p}\left(N_{0}(g)\right) \cap \widehat{\exp _{p}}\left(N_{0}(\hat{g})\right) \subset M,
$$

and since $\exp _{p}$ and $\widehat{\exp }_{p}$ are local diffeomorphisms from $N_{0}(g) \cap N_{0}(\hat{g}) \subset T_{p} M$ into $M_{p}$, the map defined by

$$
\begin{aligned}
\psi:\left(M_{p} \cap \mathcal{C}_{L}^{-}(p, \hat{g}), \hat{g}\right) & \longrightarrow\left(M_{p} \cap \mathcal{C}_{L}^{-}(p, g), g\right) \\
\hat{q} & \longmapsto \psi(\hat{q})=q=\exp _{p}\left(\widehat{\exp }_{p}^{-1}(\hat{q})\right)
\end{aligned}
$$

is a diffeomorphism with $\psi(p)=\operatorname{id}_{M}$. In particular, in terms of the coordinates $\left\{x^{i}\right\}$ and $\left\{y^{a}\right\}$ we can locally write

$$
y^{a}(\hat{q}) \longmapsto x^{i}(q)=\psi^{i}\left(y^{b}(\hat{q})\right) .
$$

In order to describe at a given length scale $0<L \leq L_{0}$, the effect of these diffeomorphisms on the lightcone regions $\mathcal{C}_{L}^{-}(p, g)$ and $\mathcal{C}_{L}^{-}(p, \hat{g})$, let us consider the spherical surfaces

$$
\left(\Sigma_{L}, h\right):=[\Sigma(p, r=L), h], \quad\left(\widehat{\Sigma}_{L}, \hat{h}\right):=[\widehat{\Sigma}(p, \hat{r}=L), \hat{h}]
$$

with their respective metrics $h$ and $\hat{h}$, and where, since the notation wants to travel light, we drop the explicit reference to the vertex $p$ of the lightcone and where we have replaced the affine parameters $r$ and $\hat{r}$ with the preassigned value $L$ of the probed length scale. The surfaces $\left(\Sigma_{L}, h\right)$ and $\left(\widehat{\Sigma}_{L}, \hat{h}\right)$ characterize, at the given scale $L$, the celestial sphere at $p$ as seen by the phenomenological observer and by the reference FLRW observer, respectively.

A direct application of the standard geometrical setup of harmonic map theory (see, e.g., [22]) provides the following notational lemma directly connecting our analysis to harmonic maps between surfaces.

\footnotetext{
5 We use the letters from the first half of the alphabet, $a, b, c, d, \ldots$ to index the coordinates $\{y\}$; the letters from the second half $i, j, k, \ell, \ldots$ provide the indexing of the coordinates $\{x\}$.
} 
Lemma 4 Let $\psi_{L}$ be the diffeomorphism $\psi$ restricted to the surfaces $\left(\widehat{\Sigma}_{L}, \hat{h}\right)$ and $\left(\Sigma_{L}, h\right)$

$$
\psi_{L}:\left(\widehat{\Sigma}_{L}, \hat{h}\right) \longrightarrow\left(\Sigma_{L}, h\right)
$$

then we can introduce the pullback bundle $\psi_{L}^{-1} T \widehat{\Sigma}_{L}$ whose sections $v \equiv \psi_{L}^{-1} V:=$ $V \circ \psi_{L}, V \in C^{\infty}\left(\widehat{\Sigma}, T \widehat{\Sigma}_{L}\right)$, are the vector fields over $\widehat{\Sigma}$ covering the map $\psi_{L}$. If $T^{*} \widehat{\Sigma}_{L}$ denotes the cotangent bundle to $\left(\widehat{\Sigma}_{L}, \hat{h}\right)$, then the differential $d \psi_{L}=\frac{\partial \psi_{L}^{i}}{\partial y^{a}} d y^{a} \otimes \frac{\partial}{\partial \psi^{i}}$ can be interpreted as a section of $T^{*} \widehat{\Sigma}_{L} \otimes \psi^{-1} T \Sigma_{L}$, and its Hilbert-Schmidt norm, in the bundle metric

$$
\langle\cdot, \cdot\rangle_{T * \widehat{\Sigma}_{L} \otimes \psi^{-1} T \Sigma_{L}}:=\hat{h}^{-1}(y) \otimes h\left(\psi_{L}(y)\right)(\cdot, \cdot),
$$

is provided by

$$
\left\langle d \psi_{L}, d \psi_{L}\right\rangle_{T^{*} \widehat{\Sigma}_{L} \otimes \psi^{-1} T \Sigma_{L}}=\hat{h}^{a b}(x) \frac{\partial \psi^{i}(y)}{\partial y^{a}} \frac{\partial \psi^{j}(y)}{\partial y^{b}} h_{i j}(\psi(y))=\operatorname{tr}_{\hat{h}(y)}\left(\psi_{L}^{*} h\right),
$$

where

$$
\psi_{L}^{*} h \Longrightarrow\left(\psi_{L}^{*} h\right)_{a b}=\frac{\partial \psi^{i}\left(y^{c}\right)}{\partial y^{a}} \frac{\partial \psi^{k}\left(y^{d}\right)}{\partial y^{b}} h_{i k}
$$

provides the pullback of the metric $h$ on $\widehat{\Sigma}_{L}$.

The connection between the pulled-back metric $\psi_{L}^{*} h$ and the round metric $\hat{h}$, both defined on $\widehat{\Sigma}_{L}$, is provided by the following proposition where we, respectively, denote by $R_{L}(\hat{h})$ and $R_{L}(h)$ the scalar curvature of $\left(\widehat{\Sigma}_{L}, \hat{h}\right)$ and $\left(\Sigma_{L}, h\right)$, and we let $\Delta_{\hat{h}}:=\hat{h}^{\alpha \beta} \nabla_{\alpha} \nabla_{\beta}$ be the Laplace-Beltrami operator on $\left(\widehat{\Sigma}_{L}, \hat{h}\right)$. Notice that the scalar curvature $R_{L}(\hat{h})$ is associated with the metric (17) evaluated for $\hat{r}=L$ and hence is given by the constant $R_{L}(\hat{h})=\frac{2}{a^{2}(L) L^{2}}$. In a similar way, $R_{L}(h)$ is associated with the metric (12) evaluated for $r=L$, and as such it depends on the area distance $D^{2}(L)$ and on the lensing distortion $\mathcal{L}_{\alpha \beta}(L) \mathrm{d} x^{\alpha} \mathrm{d} x^{\beta}$.

Proposition 5 Let $q_{(i)}, i=1,2,3$ three distinct points intercepted, on the observer celestial sphere $\left(\Sigma_{L}, h\right)$, by three past-directed null geodesics on $\mathcal{C}_{L}^{-}(p, g)$, and let $\hat{q}_{(i)}, i=1,2,3$, three distinguished points on the reference FLRW celestial sphere $\left(\widehat{\Sigma}_{L}, \hat{h}\right)$, characterizing three corresponding past-directed null directions on $\mathcal{C}_{L}^{-}(p, \hat{g})$. If $\zeta \in \operatorname{PSL}(2, \mathbb{C})$ denotes the fractional linear transformation in the projective special linear group, describing the automorphism of $\left(\widehat{\Sigma}_{L}, \hat{h}\right)$ that brings $\left\{\psi^{-1}\left(q_{i}\right)\right\}$ into $\hat{q}_{(i)}, i=1,2,3$, then there is a positive scalar function $\Phi_{\widehat{\Sigma} \Sigma} \in C^{\infty}(\hat{\Sigma}, \mathbb{R})$, solution of the elliptic partial differential equation

$$
-\Delta_{\hat{h}} \ln \left(\Phi_{\widehat{\Sigma} \Sigma}^{2}\right)+R_{L}(\hat{h})=R_{L}(h) \Phi_{\widehat{\Sigma} \Sigma}^{2},
$$

such that $\psi_{L} \circ \zeta$ characterizes a conformal diffeomorphism between $\left(\widehat{\Sigma}_{L}, \hat{h}\right)$ and $\left(\Sigma_{L}, h\right)$, i.e.,

$$
\left(\psi_{L} \circ \zeta\right)^{*} h=\Phi_{\widehat{\Sigma} \Sigma}^{2} \hat{h}
$$


Proof This is a direct consequence of the Poincare-Koebe uniformization theorem which implies that the 2 -sphere with the pulled back metric $\left(\widehat{\Sigma}_{L}, \psi_{L}^{*} h\right)$ can be mapped conformally, in a one-to-one way, onto the round 2-sphere $\left(\widehat{\Sigma}_{L}, \hat{h}\right)$. Recall that on the unit sphere $\mathbb{S}^{2}$, with its canonical round metric $\mathrm{d} \Omega^{2}$, there is a unique conformal class $\left[\mathrm{d} \Omega^{2}\right]$ and that the metric (17) on $\widehat{\Sigma}_{L} \simeq \mathbb{S}^{2}$, rescaled according to $\hat{h} /\left(a^{2}(\hat{r}) \hat{r}^{2}\right)$, is isometric to $\mathrm{d} \Omega^{2}$. Hence, by the uniformization theorem, all metrics on $\widehat{\Sigma}_{L} \simeq \mathbb{S}^{2}$ may be pulled back by conformal diffeomorphisms to the conformal class $[\hat{h}]$ of the round metric with the chosen radius $a^{2}(\hat{r}) \hat{r}^{2}$. Since $\left(\widehat{\Sigma}_{L}, \hat{h} /\left(a^{2}(\hat{r}) \hat{r}^{2}\right)\right) \simeq \mathbb{S}^{2}$, the pullback is unique modulo the action of the conformal group of the sphere $\operatorname{Conf}\left(\mathbb{S}^{2}\right)$. If we denote by $\mathcal{P}_{\mathbb{S}^{2}}$ the stereographic projection (from the north pole $(0,0,1)$ of $\left.\mathbb{S}^{2}:=\left\{(x, y, z) \in \mathbb{R}^{3} \mid x^{2}+y^{2}+z^{2}=1\right\}\right)$

$$
\mathcal{P}_{\mathbb{S}^{2}}: \mathbb{S}^{2} \subset \mathbb{R}^{3} \longrightarrow \mathbb{C} \cup\{\infty\}, \mathcal{P}_{\mathbb{S}^{2}}(x, y, z)=\frac{x+i y}{1-z},
$$

then we can identify Conf $\left(\mathbb{S}^{2}\right)$ with the six-dimensional projective special linear group $\operatorname{PSL}(2, \mathbb{C})$ describing the automorphisms of $\mathbb{S}^{2} \simeq \mathbb{C} \cup\{\infty\}$. The elements of PSL $(2, \mathbb{C})$ are the fractional linear transformations the Riemann sphere $\mathbb{S}^{2} \simeq \mathbb{C} \cup\{\infty\}$

$$
\begin{aligned}
\mathbb{C} \cup\{\infty\} & \longrightarrow \mathbb{C} \cup\{\infty\} \\
z & \longmapsto \zeta(z):=\frac{a z+b}{c z+d}, \quad a, b, c, d \in \mathbb{C}, \quad a d-b c \neq 0 .
\end{aligned}
$$

These transformations act on the diffeomorphism (27) according to

$$
\begin{aligned}
\operatorname{PSL}(2, \mathbb{C}) \times\left(\widehat{\Sigma}_{L}, \hat{h}\right) & \longrightarrow\left(\Sigma_{L}, h\right) \\
(\zeta, y) & \longmapsto \psi_{L}(\zeta(y))
\end{aligned}
$$

where, abusing notation, we have denoted by $\zeta(y)$ the action that the fractional linear transformation $\zeta(z)$ defines on the point $y \in \widehat{\Sigma}_{L}$ corresponding, via stereographic projection, to the point $z \in \mathbb{C} \cup\{\infty\}$. This action may be a potential source of a delicate problem since $\operatorname{PSL}(2, \mathbb{C})$ is non-compact and $\Phi_{\widehat{\Sigma} \Sigma}$ is evaluated on the composition $\psi_{L} \circ \zeta$ defined by (35). This is not problematic as long as $\zeta$ varies in the maximal compact subgroup of $\operatorname{PSL}(2, \mathbb{C})$ generated by the isometries of $(\widehat{\Sigma}, \hat{h})$. However, if we consider a sequence $\left\{\zeta_{k}\right\}_{k \in \mathbb{N}} \in P S L(2, \mathbb{C})$ defined by larger and larger dilation (corresponding to larger and larger (local) Lorentz boosts of the surface $\widehat{\Sigma}$ in the reference spacetime $(M, \hat{g}))$, then the composition $\psi_{L} \circ \zeta_{k}$ may generate a sequence of conformal factors $\left\{\Phi_{(k)}^{2} \widehat{\Sigma} \Sigma\right.$ \} converging to a non-smooth function. To avoid these pathologies, we exploit the fact that a linear fractional transformation is fully determined if we fix its action on three distinct points of the sphere. In our setting, this corresponds to fixing the action on three distinct null direction in the lightcone region $\mathcal{C}_{L}^{-}(p, \hat{g})$. In physical terms this is equivalent to require that the FLRW reference observer at $p$ has to adjust his velocity and orientation in such a way that three given astrophysical sources of choice are in three specified position on the celestial sphere $\left(\widehat{\Sigma}_{L}, \hat{h}\right)$ at scale $L$. This is a gauge fixing of the action of $\operatorname{PSL}(2, \mathbb{C})$ that corresponds in a very natural way to adjust the location of three reference observations in order to 
be able to compare the data on the phenomenological past lightcone $\mathcal{C}_{L}^{-}(p, g)$ with the data on the reference past lightcone $\mathcal{C}_{L}^{-}(p, \hat{g})$. By fixing in this way the PSL $(2, \mathbb{C})$ action, the pullback $\left(\psi_{L} \circ \zeta\right)^{*} h$ on $\hat{\Sigma}_{L}$ of the metric $h$ is well defined. By the PoincareKoebe uniformization theorem, the metric $\left(\psi_{L} \circ \zeta\right)^{*} h$ is in the same conformal class of $\hat{h}$. Let us denote by $\Phi_{\widehat{\Sigma} \Sigma}^{2} \in C^{\infty}(\hat{\Sigma}, \mathbb{R})$ the corresponding conformal factor such that $\left(\psi_{L} \circ \zeta\right)^{*} h=\Phi_{\widehat{\Sigma} \Sigma}^{2} \hat{h}$. If we set $e^{f}:=\Phi_{\widehat{\Sigma} \Sigma}^{2}$, then the properties of the scalar curvature under the conformal transformation $h=e^{f} \hat{h}$ (see, e.g., [1]) provide the relation

$$
R\left(\left(\psi_{L} \circ \zeta\right)^{*} h\right)=e^{-f}\left[R(\hat{h})+\Delta_{\hat{h}} f\right] .
$$

If for notational ease we keep on writing $R(h)$ for $R\left(\left(\psi_{L} \circ \zeta\right)^{*} h\right)=R\left(h\left(\psi_{L} \circ \zeta\right)\right)$, then it follows from (36) that $\Phi_{\widehat{\Sigma}}^{2}$ necessarily is a solution on $\left(\widehat{\Sigma}_{L}, \hat{h}\right)$ of the elliptic partial differential equation (31), solution that under the stated hypotheses always exists [1].

According to the above result, there is a positive scalar function $\Phi_{\widehat{\Sigma} \Sigma} \in C^{\infty}(\hat{\Sigma}, \mathbb{R})$ such that $\psi_{L} \circ \zeta$ characterizes a conformal diffeomorphism between $\left(\hat{\Sigma}_{L}, \hat{h}\right)$ and $\left(\Sigma_{L}, h\right)$. In components (32) can be written as

$$
\left(\left(\psi_{L} \circ \zeta\right)^{*} h\right)_{a b}=\frac{\partial \psi_{L}^{i}(\zeta(y))}{\partial y^{a}} \frac{\partial \psi_{L}^{k}(\zeta(y))}{\partial y^{b}} h_{i k}=\Phi_{\widehat{\Sigma} \Sigma}^{2} \hat{h}_{a b}
$$

It follows that by tracing (37) with respect to $\hat{h}^{a b}$, we can express $\Phi_{\widehat{\Sigma} \Sigma}^{2}$ in terms of the Hilbert-Schmidt norm of the differential $d\left(\psi_{L} \circ \zeta\right)=\frac{\partial \psi_{L}^{i}(\zeta(y))}{\partial y^{a}} \mathrm{~d} y^{a} \otimes \frac{\partial}{\partial \psi_{L}^{i}}$ according to $($ see $(29))$

$$
\Phi_{\widehat{\Sigma} \Sigma}^{2}=t r_{\hat{h}(y)}\left(\left(\psi_{L} \circ \zeta\right)^{*} h\right)=\frac{1}{2} \hat{h}^{a b} \frac{\partial \psi_{L}^{i}(\zeta(y))}{\partial y^{a}} \frac{\partial \psi_{L}^{k}(\zeta(y))}{\partial y^{b}} h_{i k}
$$

From (37) we get det $\left(\left(\psi_{L} \circ \zeta\right)^{*} h\right)=\Phi_{\widehat{\Sigma} \Sigma}^{4} \operatorname{det}(\hat{h})$; hence, we can equivalently write the conformal factor as the Radon-Nikodym derivative of the Riemannian measure $\mathrm{d} \mu_{\psi^{*} h}:=\left(\psi_{L} \circ \zeta\right)^{*} \mathrm{~d} \mu$, of $\left(\hat{\Sigma},\left(\psi_{L} \circ \zeta\right)^{*} h\right)$, with respect to the Riemannian measure $\mathrm{d} \mu_{\hat{h}}$ of the round metric $(\hat{\Sigma}, \hat{h})$, i.e.,

$$
\Phi_{\widehat{\Sigma} \Sigma}^{2}=\frac{\mathrm{d} \mu_{\psi^{*} h}}{\mathrm{~d} \mu_{\hat{h}}}=\frac{\left(\psi_{L} \circ \zeta\right)^{*} \mathrm{~d} \mu_{h}}{\mathrm{~d} \mu_{\hat{h}}}
$$

Equivalently, this states that $\Phi_{\widehat{\Sigma} \Sigma}^{2}$ can be interpreted as the Jacobian of the map $\psi_{L} \circ \zeta$,

$$
\Phi_{\widehat{\Sigma} \Sigma}^{2}=\operatorname{Jac}\left(\psi_{L} \circ \zeta\right)
$$

Along the same lines, we can associate with the inverse diffeomorphism

$$
\left(\psi_{L} \circ \zeta\right)^{-1}:\left(\Sigma_{L}, h\right) \longrightarrow\left(\widehat{\Sigma}_{L}, \hat{h}\right)
$$




$$
x \longmapsto \zeta^{-1}\left(\psi_{L}^{-1}(x)\right)
$$

a positive scalar function $\Phi_{\Sigma \widehat{\Sigma}} \in C^{\infty}(\Sigma, \mathbb{R})$ such that we can write

$$
\left(\left(\psi_{L} \circ \zeta\right)^{-1}\right)^{*} \hat{h}=\Phi_{\Sigma \widehat{\Sigma}}^{2} h
$$

with

$$
\begin{aligned}
\Phi_{\Sigma \widehat{\Sigma}}^{2} & =\frac{1}{2} h^{i k} \frac{\partial\left(\zeta^{-1}\left(\psi_{L}^{-1}(x)\right)\right)^{a}}{\partial x^{i}} \frac{\partial\left(\zeta^{-1}\left(\psi_{L}^{-1}(x)\right)\right)^{b}}{\partial x^{k}} \hat{h}_{a b}=\frac{\mathrm{d} \mu_{\left(\psi^{-1}\right) * \hat{h}}}{\mathrm{~d} \mu_{h}} \\
& =\frac{\left(\left(\psi_{L} \circ \zeta\right)^{-1}\right)^{*} \mathrm{~d} \mu_{\hat{h}}}{\mathrm{~d} \mu_{h}} .
\end{aligned}
$$

To measure the global deviation of the conformal diffeomorphisms $\Phi_{\widehat{\Sigma} \Sigma}$ from an isometry between $\left(\hat{\Sigma}_{L}, \hat{h}\right)$ and $\left(\Sigma_{L}, h\right)$, we introduce the following comparison functional where, for later use, we keep track of the $\zeta \in \operatorname{PSL}(2, \mathbb{C})$ dependence in $\Phi_{\widehat{\Sigma} \Sigma}$.

Definition 6 (The lightcone comparison functional at scale L) Let $\Phi_{\widehat{\Sigma} \Sigma} \in$ $C^{\infty}(\hat{\Sigma}, \mathbb{R})$ (or at least $C^{2}(\hat{\Sigma}, \mathbb{R})$ ) be the positive scalar function such that $\psi_{L} \circ \zeta$ characterizes the conformal diffeomorphism $\left(\psi_{L} \circ \zeta\right)^{*} h=\Phi_{\widehat{\Sigma} \Sigma}^{2} \hat{h}$ between $\left(\widehat{\Sigma}_{L}, \hat{h}\right)$ and $\left(\Sigma_{L}, h\right)$, then the associated lightcone comparison functional at scale $L$ is defined by

$$
E_{\widehat{\Sigma} \Sigma}\left[\psi_{L}, \zeta\right]:=\int_{\widehat{\Sigma}_{L}}\left(\Phi_{\widehat{\Sigma} \Sigma}-1\right)^{2} \mathrm{~d} \mu_{\hat{h}} .
$$

The functional $E_{\widehat{\Sigma} \Sigma}\left[\psi_{L}, \zeta\right]$ is clearly related to the familiar harmonic map energy associated with the map $\psi_{L} \circ \zeta: \hat{\Sigma} \longrightarrow \Sigma$. Explicitly, if we take into account (38), we can write

$$
\int_{\widehat{\Sigma}_{L}} \widehat{\Phi}_{L}^{2} \mathrm{~d} \mu_{\hat{h}}=\frac{1}{2} \int_{\widehat{\Sigma}_{L}} \hat{h}^{a b} \frac{\partial \psi_{L}^{i}(\zeta(y))}{\partial y^{a}} \frac{\partial \psi_{L}^{k}(\zeta(y))}{\partial y^{b}} h_{i k} \mathrm{~d} \mu_{\hat{h}}
$$

which provides the harmonic map functional whose critical point is the harmonic maps of the Riemann surface $\left(\hat{\Sigma}_{L},[\hat{h}]\right)$ into $\left(\Sigma_{L}, h\right)$, where $[\hat{h}]$ denotes the conformal class of the metric $\hat{h}$. Notice that whereas the harmonic map energy (45) is a conformal invariant quantity, the functional $E_{\widehat{\Sigma} \Sigma}\left[\psi_{L}, \zeta\right]$ is not conformally invariant. Under a conformal transformation $\hat{h} \longrightarrow e^{2 f} \hat{h}$, we get

$$
\int_{\widehat{\Sigma}_{L}}\left(e^{-f} \Phi_{\widehat{\Sigma} \Sigma}-1\right)^{2} e^{2 f} \mathrm{~d} \mu_{\hat{h}}
$$

It is also clear from its definition that corresponding to large gradients (see (43)), $E_{\widehat{\Sigma} \Sigma}\left[\psi_{L}, \zeta\right]$ tends to the harmonic map energy. In this connection, it is important 
to stress that rather than on the space of smooth maps $C^{\infty}(\widehat{\Sigma}, \Sigma)$, the functional $E_{\widehat{\Sigma} \Sigma}\left[\psi_{L}, \zeta\right]$ is naturally defined on the Sobolev space of maps $W^{1,2}(\widehat{\Sigma}, \Sigma)$ which are, together with their weak derivatives, square integrable. This characterization, familiar when studying weakly harmonic maps [19] and which we discuss in detail below when minimizing $E_{\widehat{\Sigma} \Sigma}\left[\psi_{L}, \zeta\right]$, is important in our case when extending our analysis to the low regularity setting when lightcone caustics are present.

Remark 7 It must be stressed that energy functionals such as (44) are rather familiar in the problem of comparing shapes of surfaces in relation with computer graphic and visualization problems (see, e.g., [16,20], to quote two relevant papers in a vast literature). In particular, (44) has been introduced under the name of elastic energy in an inspiring paper by Hass and Koehl [18], who use it as a building block of a more complex functional relevant to surface visualization.

In our particular framework, the functional $E_{\widehat{\Sigma} \Sigma}\left[\psi_{L}, \zeta\right]$ has a number of important properties that make it a natural candidate for comparing, at the given length scale $L$, the physical lightcone region $\mathcal{C}_{L}^{-}(p, g)$ with the FLRW reference region $\mathcal{C}_{L}^{-}(p, \hat{g})$. To start with, we prove the following general properties (in the smooth setting).

Lemma 8 The functional $E_{\widehat{\Sigma} \Sigma}\left[\psi_{L}, \zeta\right]$ is symmetric

$$
E_{\widehat{\Sigma} \Sigma}\left[\psi_{L}, \zeta\right]=E_{\Sigma \widehat{\Sigma}}\left[\psi_{L}^{-1}, \zeta^{-1}\right]
$$

where

$$
E_{\Sigma \widehat{\Sigma}}\left[\psi_{L}^{-1}, \zeta^{-1}\right]:=\int_{\Sigma_{L}}\left(\Phi_{\Sigma \widehat{\Sigma}}-1\right)^{2} d \mu_{h}
$$

is the comparison functional associated with the inverse map $\left(\psi_{L} \circ \zeta\right)^{-1}: \Sigma_{L} \longrightarrow$ $\hat{\Sigma}_{L}$

If $\left(\dot{\widetilde{\Sigma}}_{L}, \tilde{h}\right)$ is a third surface on the past lightcone $\widetilde{\mathcal{C}}_{L_{0}}^{-}(p, \tilde{g})$, with vertex at $p$, associated with yet another reference FLRW metric $\tilde{g}$ on $M$ (say another member of the FLRW family of spacetimes, distinct from $\hat{g}$ ), and $\sigma_{L}: \Sigma_{L} \longmapsto \widetilde{\Sigma}_{L}, \Phi_{\Sigma} \widetilde{\Sigma}$, respectively, are the corresponding diffeomorphism and conformal factor, then to the composition of maps

$$
\widehat{\Sigma}_{L} \underset{\psi_{L}}{\longrightarrow} \Sigma_{L} \underset{\sigma_{L}}{\longrightarrow} \widetilde{\Sigma}_{L}
$$

we can associate the triangular inequality

$$
E_{\widehat{\Sigma} \Sigma}\left[\psi_{L}, \zeta\right]+E_{\Sigma \widetilde{\Sigma}}\left[\sigma_{L}, \zeta\right] \geq E_{\widehat{\Sigma} \widetilde{\Sigma}}\left[\left(\sigma_{L} \circ \psi_{L}\right), \zeta\right]
$$

where

$$
E_{\widehat{\Sigma} \tilde{\Sigma}}\left[\left(\sigma_{L} \circ \psi_{L}\right), \zeta\right]:=\int_{\hat{\Sigma}_{L}}\left(\Phi_{\widehat{\Sigma} \widetilde{\Sigma}}-1\right)^{2} d \mu_{\hat{h}} .
$$

If $A\left(\widehat{\Sigma}_{L}\right):=\int_{\widehat{\Sigma}_{L}} d \mu_{\hat{h}}$ and $A\left(\Sigma_{L}\right):=\int_{\Sigma_{L}} d \mu_{h}$, respectively, denote the area of the surfaces $(\widehat{\Sigma}, \hat{h})$ and $(\Sigma, h)$, then we have the upper and lower bounds

$$
A\left(\widehat{\Sigma}_{L}\right)+A\left(\Sigma_{L}\right) \geq E_{\widehat{\Sigma} \Sigma}\left[\psi_{L}, \zeta\right] \geq\left(\sqrt{\mathcal{A}\left(\widehat{\Sigma}_{L}\right)}-\sqrt{A\left(\Sigma_{L}\right)}\right)^{2} .
$$


Finally,

$$
E_{\widehat{\Sigma} \Sigma}\left[\psi_{L}, \zeta\right]=0
$$

iff the surfaces $(\widehat{\Sigma}, \hat{h})$ and $(\Sigma, h)$ are isometric.

Proof For notational ease, let us temporarily dismiss the action of the linear fractional transformation $\zeta \in \operatorname{PSL}(2, \mathbb{C})$ and, if there is no chance of confusion, write $E_{\widehat{\Sigma} \Sigma}\left[\psi_{L}\right]$ in place of the full $E_{\widehat{\Sigma} \Sigma}\left[\psi_{L}, \zeta\right]$. We start with proving the symmetry property (47). To this end, expand the integrand in (44) and rewrite $E_{\widehat{\Sigma} \Sigma}\left[\psi_{L}\right]$ as

$$
\begin{aligned}
E_{\widehat{\Sigma} \Sigma}\left[\psi_{L}\right] & =\int_{\widehat{\Sigma}_{L}}\left(\Phi_{\widehat{\Sigma} \Sigma}-1\right)^{2} \mathrm{~d} \mu_{\hat{h}}=\int_{\widehat{\Sigma}_{L}} \Phi_{\widehat{\Sigma} \Sigma}^{2} \mathrm{~d} \mu_{\hat{h}}+\int_{\widehat{\Sigma}_{L}} \mathrm{~d} \mu_{\hat{h}}-2 \int_{\widehat{\Sigma}_{L}} \Phi_{\widehat{\Sigma} \Sigma} \mathrm{d} \mu_{\hat{h}} \\
& =\int_{\widehat{\Sigma}_{L}} \frac{\psi_{L}^{*} \mathrm{~d} \mu_{h}}{\mathrm{~d} \mu_{\hat{h}}} \mathrm{~d} \mu_{\hat{h}}+A\left(\widehat{\Sigma}_{L}\right)-2 \int_{\widehat{\Sigma}_{L}} \Phi_{\widehat{\Sigma} \Sigma} \mathrm{d} \mu_{\hat{h}} \\
& =\int_{\psi_{L}\left(\widehat{\Sigma}_{L}\right)} \mathrm{d} \mu_{h}+A\left(\widehat{\Sigma}_{L}\right)-2 \int_{\widehat{\Sigma}_{L}} \Phi_{\widehat{\Sigma} \Sigma} \mathrm{d} \mu_{\hat{h}} \\
& =A\left(\Sigma_{L}\right)+A\left(\widehat{\Sigma}_{L}\right)-2 \int_{\widehat{\Sigma}_{L}} \Phi_{\widehat{\Sigma} \Sigma} \mathrm{d} \mu_{\hat{h}}
\end{aligned}
$$

where we have exploited the Radon-Nikodym characterization of $\widehat{\Phi}_{\widehat{\Sigma} \Sigma}^{2}$, (see (39)), the identification $\psi\left(\widehat{\Sigma}_{L}\right)=\Sigma_{L}$, and the relation

$$
\int_{\widehat{\Sigma}_{L}} \frac{\psi_{L}^{*} \mathrm{~d} \mu_{h}}{\mathrm{~d} \mu_{\hat{h}}} \mathrm{~d} \mu_{\hat{h}}=\int_{\widehat{\Sigma}_{L}} \psi_{L}^{*} \mathrm{~d} \mu_{h}=\int_{\psi\left(\widehat{\Sigma}_{L}\right)} \mathrm{d} \mu_{h}=\int_{\Sigma_{L}} \mathrm{~d} \mu_{h}=A\left(\Sigma_{L}\right),
$$

where $A\left(\Sigma_{L}\right)$ and $A\left(\widehat{\Sigma}_{L}\right)$, respectively, denote the area of $\left(\hat{\Sigma}_{L}, \hat{h}\right)$ and $\left(\Sigma_{L}, h\right)$. Along the same lines, let us compute the lightcone comparison functional $E_{\Sigma \widehat{\Sigma}}\left[\psi_{L}^{-1}\right]$ associated with the inverse diffeomorphism $\psi_{L}^{-1}:\left(\Sigma_{L}, h\right) \longrightarrow\left(\hat{\Sigma}_{L}, \hat{h}\right)$ and the corresponding conformal factor $\Phi_{\Sigma \widehat{\Sigma}} \in C^{\infty}(\Sigma, \mathbb{R})$ - (see (42)),

$$
E_{\Sigma \widehat{\Sigma}}\left[\psi_{L}^{-1}\right]:=\int_{\Sigma_{L}}\left(\Phi_{\Sigma \widehat{\Sigma}}-1\right)^{2} \mathrm{~d} \mu_{h}
$$

We have

$$
E_{\Sigma \widehat{\Sigma}}\left[\psi_{L}^{-1}\right]:=A\left(\widehat{\Sigma}_{L}\right)+A\left(\Sigma_{L}\right)-2 \int_{\Sigma_{L}} \Phi_{\Sigma \widehat{\Sigma}} \mathrm{d} \mu_{h}
$$

Since

$$
\begin{aligned}
\int_{\Sigma_{L}} \Phi_{\Sigma \widehat{\Sigma}} \mathrm{d} \mu_{h} & =\int_{\Sigma_{L}} \sqrt{\frac{\mathrm{d} \mu_{\left(\psi^{-1}\right)^{*} \hat{h}}}{\mathrm{~d} \mu_{h}}} \mathrm{~d} \mu_{h}=\int_{\Sigma_{L}} \sqrt{\frac{\mathrm{d} \mu_{\left(\psi^{-1}\right)^{*} \hat{h}}}{\mathrm{~d} \mu_{h}}} \frac{\mathrm{d} \mu_{h}}{\mathrm{~d} \mu_{\left(\psi^{-1}\right)^{*} \hat{h}}} \mathrm{~d} \mu_{\left(\psi^{-1}\right)^{*} \hat{h}} \\
& =\int_{\Sigma_{L}} \sqrt{\frac{\mathrm{d} \mu_{h}}{\mathrm{~d} \mu_{\left(\psi^{-1}\right)^{*} \hat{h}}}}\left(\psi^{-1}\right)^{*} \mathrm{~d} \mu_{\hat{h}}
\end{aligned}
$$


On the other hand, if we take the pullback, under the action of $\psi_{L}^{-1}:\left(\Sigma_{L}, h\right) \longrightarrow$ $\left(\widehat{\Sigma}_{L}, \hat{h}\right)$, of the relation $\Phi_{\widehat{\Sigma} \Sigma}^{2} \mathrm{~d} \mu_{\hat{h}}=\psi_{L}^{*} \mathrm{~d} \mu_{h}$, (see (39)), we have

$$
\begin{aligned}
\left(\psi^{-1}\right)^{*}\left(\Phi_{\widehat{\Sigma} \Sigma}^{2} \mathrm{~d} \mu_{\hat{h}}\right) & =\left(\psi_{L}^{-1}\right)^{*}\left(\psi_{L}^{*} \mathrm{~d} \mu_{h}\right) \\
& \Longrightarrow \Phi_{\widehat{\Sigma} \Sigma}^{2}\left(\psi_{L}^{-1}(x)\right)\left(\left(\psi_{L}^{-1}\right)^{*} \mathrm{~d} \mu_{\hat{h}}\right)(x)=\mathrm{d} \mu_{h}(x),
\end{aligned}
$$

from which we get

$$
\Phi_{\widehat{\Sigma} \Sigma}^{2}\left(\psi_{L}^{-1}(x)\right)=\frac{\mathrm{d} \mu_{h}(x)}{\left(\left(\psi_{L}^{-1}\right)^{*} \mathrm{~d} \mu_{\hat{h}}\right)(x)} .
$$

Hence, we can rewrite (58) as

$$
\begin{aligned}
\int_{\Sigma_{L}} \Phi_{\Sigma \widehat{\Sigma}} \mathrm{d} \mu_{h} & =\int_{\Sigma_{L}} \sqrt{\frac{\mathrm{d} \mu_{h}}{\mathrm{~d} \mu_{\left(\psi^{-1}\right) * \hat{h}}}}\left(\psi_{L}^{-1}\right)^{*} \mathrm{~d} \mu_{\hat{h}}=\int_{\Sigma_{L}} \Phi_{\widehat{\Sigma} \Sigma}\left(\psi_{L}^{-1}\right)\left(\psi_{L}^{-1}\right)^{*} \mathrm{~d} \mu_{\hat{h}} \\
& =\int_{\Sigma_{L}}\left(\psi_{L}^{-1}\right)^{*}\left(\Phi_{\widehat{\Sigma} \Sigma} \mathrm{d} \mu_{\hat{h}}\right)=\int_{\psi^{-1}\left(\Sigma_{L}\right)} \Phi_{\widehat{\Sigma} \Sigma} \mathrm{d} \mu_{\hat{h}} \\
& =\int_{\hat{\Sigma}_{L}} \Phi_{\widehat{\Sigma} \Sigma} \mathrm{d} \mu_{\hat{h}}
\end{aligned}
$$

and

$$
\begin{aligned}
E_{\Sigma \widehat{\Sigma}}\left[\psi^{-1}\right]: & =A\left(\widehat{\Sigma}_{L}\right)+A\left(\Sigma_{L}\right)-2 \int_{\Sigma_{L}} \Phi_{\Sigma \widehat{\Sigma}} \mathrm{d} \mu_{h} \\
& =A\left(\widehat{\Sigma}_{L}\right)+A\left(\Sigma_{L}\right)-2 \int_{\hat{\Sigma}_{L}} \Phi_{\widehat{\Sigma} \Sigma} \mathrm{d} \mu_{\hat{h}}=E_{\widehat{\Sigma} \Sigma}[\psi]
\end{aligned}
$$

Hence, the comparison functional is symmetric.

In order to prove the triangular inequality (50), let us consider the sum

$$
E_{\widehat{\Sigma} \Sigma}\left[\psi_{L}\right]+E_{\Sigma \widetilde{\Sigma}}\left[\sigma_{L}\right]=\int_{\hat{\Sigma}_{L}}\left(\Phi_{\widehat{\Sigma} \Sigma}-1\right)^{2} \mathrm{~d} \mu_{\hat{h}}+\int_{\Sigma_{L}}\left(\Phi_{\Sigma \widetilde{\Sigma}}-1\right)^{2} \mathrm{~d} \mu_{h}
$$

From the relation (59), we have $\mathrm{d} \mu_{h}=\Phi_{\widehat{\Sigma} \Sigma}^{2}\left(\psi_{L}^{-1}\right)\left(\psi_{L}^{-1}\right)^{*} \mathrm{~d} \mu_{\hat{h}}$, and we can write

$$
\int_{\Sigma_{L}}\left(\Phi_{\Sigma \widetilde{\Sigma}}-1\right)^{2} \mathrm{~d} \mu_{h}=\int_{\widehat{\Sigma}_{L}}\left(\Phi_{\Sigma \widetilde{\Sigma}}-1\right)^{2} \Phi_{\widehat{\Sigma} \Sigma}^{2} \mathrm{~d} \mu_{\hat{h}}
$$

Hence,

$$
E_{\widehat{\Sigma} \Sigma}\left[\psi_{L}\right]+E_{\Sigma \widetilde{\Sigma}}\left[\sigma_{L}\right]=\int_{\widehat{\Sigma}_{L}}\left[\left(\Phi_{\widehat{\Sigma} \Sigma}-1\right)^{2}+\left(\Phi_{\Sigma \widetilde{\Sigma}}-1\right)^{2} \Phi_{\widehat{\Sigma} \Sigma}^{2}\right] \mathrm{d} \mu_{\hat{h}}
$$




$$
\begin{aligned}
& \geq \int_{\widehat{\Sigma}_{L}}\left[\left(\Phi_{\widehat{\Sigma} \Sigma}-1\right)+\left(\Phi_{\Sigma \widetilde{\Sigma}}-1\right) \Phi_{\widehat{\Sigma} \Sigma}\right]^{2} \mathrm{~d} \mu_{\hat{h}} \\
& =\int_{\widehat{\Sigma}_{L}}\left(\Phi_{\widehat{\Sigma} \Sigma} \Phi_{\Sigma \widetilde{\Sigma}}-1\right)^{2} \mathrm{~d} \mu_{\hat{h}}=E_{\widehat{\Sigma} \widetilde{\Sigma}}\left[\left(\sigma_{L} \circ \psi_{L}\right)\right],
\end{aligned}
$$

where we have exploited the relation

$$
\Phi_{\Sigma \widetilde{\Sigma}}\left(\psi_{L}\right) \Phi_{\widehat{\Sigma} \Sigma}=\Phi_{\widehat{\Sigma} \widetilde{\Sigma}}
$$

which follows from observing that the positive functions $\Phi_{\widehat{\Sigma} \widetilde{\Sigma}} \in C^{\infty}(\widehat{\Sigma}, \mathbb{R})$ and $\Phi_{\Sigma \tilde{\Sigma}} \in C^{\infty}(\Sigma, \mathbb{R})$ are such that

$$
\Phi_{\widehat{\Sigma} \widetilde{\Sigma}}^{2} \hat{h}=\left(\sigma_{L} \circ \psi_{L}\right)^{*} \tilde{h}=\psi_{L}^{*}\left(\Phi_{\Sigma \widetilde{\Sigma}}^{2} h\right)=\Phi_{\Sigma \widetilde{\Sigma}}^{2}\left(\psi_{L}\right) \psi_{L}^{*} h=\Phi_{\Sigma \widetilde{\Sigma}}^{2}\left(\psi_{L}\right) \Phi_{\widehat{\Sigma} \Sigma}^{2} \hat{h},
$$

where we have set $\Phi_{\Sigma \widetilde{\Sigma}}\left(\psi_{L}\right)=\psi_{L}^{*} \Phi_{\Sigma \tilde{\Sigma}}:=\Phi_{\Sigma \tilde{\Sigma}} \circ \psi_{L}$.

From (54) and the Schwarz inequality

$$
\int_{\widehat{\Sigma}_{L}} \Phi_{\widehat{\Sigma} \Sigma} \mathrm{d} \mu_{\hat{h}} \leq\left(\int_{\widehat{\Sigma}_{L}} \Phi_{\widehat{\Sigma} \Sigma}^{2} \mathrm{~d} \mu_{\hat{h}}\right)^{1 / 2}\left(\int_{\widehat{\Sigma}_{L}} \mathrm{~d} \mu_{\hat{h}}\right)^{1 / 2}=\sqrt{A\left(\widehat{\Sigma}_{L}\right) A\left(\Sigma_{L}\right)},
$$

we get the lower bound

$$
\begin{aligned}
E_{\widehat{\Sigma} \Sigma}\left[\psi_{L}, \zeta\right] & =A\left(\Sigma_{L}\right)+A\left(\widehat{\Sigma}_{L}\right)-2 \int_{\widehat{\Sigma}_{L}} \Phi_{\widehat{\Sigma} \Sigma} \mathrm{d} \mu_{\hat{h}} \\
& \geq A\left(\Sigma_{L}\right)+A\left(\widehat{\Sigma}_{L}\right)-2 \sqrt{A\left(\widehat{\Sigma}_{L}\right) A\left(\Sigma_{L}\right)} \\
& =\left(\sqrt{\mathcal{A}\left(\widehat{\Sigma}_{L}\right)}-\sqrt{A\left(\Sigma_{L}\right)}\right)^{2}
\end{aligned}
$$

where we have exploited (55). The upper bound in (52) easily follows from (44)

$$
\begin{aligned}
E_{\widehat{\Sigma} \Sigma}\left[\psi_{L}, \zeta\right] & :=\int_{\widehat{\Sigma}_{L}}\left(\Phi_{\widehat{\Sigma} \Sigma}-1\right)^{2} \mathrm{~d} \mu_{\hat{h}} \leq \int_{\widehat{\Sigma}_{L}} \Phi_{\widehat{\Sigma} \Sigma}^{2} \mathrm{~d} \mu_{\hat{h}}+\int_{\widehat{\Sigma}_{L}} \mathrm{~d} \mu_{\hat{h}} \\
& =A\left(\widehat{\Sigma}_{L}\right)+A\left(\Sigma_{L}\right)
\end{aligned}
$$

The proof of the last part of the lemma follows observing that the integrand in $\int_{\hat{\Sigma}_{L}}\left(\Phi_{\widehat{\Sigma} \Sigma}-1\right)^{2} \mathrm{~d} \mu_{\hat{h}}$ is non-negative and, as long as $\Phi_{\widehat{\Sigma} \Sigma}$ is a smooth function on $\left(\hat{\Sigma}_{L}, \hat{h}\right)$, the condition

$$
E_{\widehat{\Sigma} \Sigma}\left[\psi_{L}\right]=\int_{\hat{\Sigma}_{L}}\left(\Phi_{\widehat{\Sigma} \Sigma}-1\right)^{2} \mathrm{~d} \mu_{\hat{h}}=0
$$

implies $\Phi_{\widehat{\Sigma} \Sigma}=1$, hence the isometry between $\left(\hat{\Sigma}_{L}, \hat{h}\right)$ and $\left(\Sigma_{L}, h\right)$. 


\section{A scale-dependent distance functional}

The properties of the comparison functional $E_{\widehat{\Sigma} \Sigma}\left[\psi_{L}, \zeta\right]$ indicate that we can associate with it a corresponding distance functional $d_{L}\left[\widehat{\Sigma}_{L}, \Sigma_{L}\right]$. To put the characterization of this distance in perspective, let us recall that a fractional linear transformations $\zeta \in \operatorname{PSL}(2, \mathbb{C})$ is fully determined if, given three distinct points of $\widehat{\Sigma} \simeq \mathbb{S}^{2}$, we specify their images. We exploited this in Proposition 5 , where we assigned three distinct points $q_{(i)}, i=1,2,3$ on the observer celestial sphere $\left(\Sigma_{L}, h\right)$, and we fixed the action of $\operatorname{PSL}(2, \mathbb{C})$ by choosing that particular automorphism $\zeta \in \operatorname{PSL}(2, \mathbb{C})$ that identifies the inverse images $\left\{\psi^{-1}\left(q_{i}\right)\right\} \in \widehat{\Sigma}_{L}$ with three chosen points $\hat{q}_{(i)}, i=1,2,3$ on the reference FLRW celestial sphere $\left(\widehat{\Sigma}_{L}, \hat{h}\right)$. Since $E_{\widehat{\Sigma} \Sigma}\left[\psi_{L}, \zeta\right]$ is not conformally invariant, the particular choice of the automorphism $\zeta \in \operatorname{PSL}(2, \mathbb{C})$, or which is the same, the particular choice of the points $q_{(i)}, i=1,2,3$ on $\left(\Sigma_{L}, h\right)$, affects $E_{\widehat{\Sigma} \Sigma}\left[\psi_{L}, \zeta\right]$; hence, it is natural to inquire if there is a choice of the automorphism $\zeta$ that minimizes $E_{\widehat{\Sigma} \Sigma}\left[\psi_{L}, \zeta\right]$. Given the reference points $\hat{q}_{(i)}, i=1,2,3$ on $\left(\widehat{\Sigma}_{L}, \hat{h}\right)$, this optimal choice for $\zeta \in \operatorname{PSL}(2, \mathbb{C})$, say $\zeta=\zeta_{0}$, will induce the proper selection of the alignment points $q_{(i)}, i=1,2,3$ on the observer celestial sphere $\left(\Sigma_{L}, h\right)$ by setting $q_{(i)}:=\psi_{L}\left(\zeta_{0}\left(\hat{q}_{(i)}\right)\right)$. In order to characterize this optimal choice, we need to minimize $E_{\widehat{\Sigma} \Sigma}\left[\psi_{L}, \zeta\right]$ over a suitable class of functions, and a natural strategy, according to these remarks, is to keep fixed the diffeomorphism ${ }^{6} \psi_{L}$ as well the points $\hat{q}_{(i)}, i=1,2,3$ on the reference $\left(\widehat{\Sigma}_{L}, \hat{h}\right)$, and let vary in a controlled way the automorphism $\zeta \in \operatorname{PSL}(2, \mathbb{C})$, so as to minimize $E_{\widehat{\Sigma} \Sigma}\left[\psi_{L}, \zeta\right]$. We also need a slightly more general setting that will allow us to deal with celestial spheres $\left(\Sigma_{L}, h\right)$ on a lightcone region $\mathcal{C}_{L}^{-}(p, g)$ where caustics develop (hence, relaxing in a controlled way the regularity of $\psi_{L}$ allowing for exponential mappings which are no longer injective). In other words, we need to extend $\psi_{L} \circ \zeta: \widehat{\Sigma}_{L} \longrightarrow \Sigma_{L}$ to be a member of a more general space of maps which allow for the low regularity setting associated with the possible presence of (isolated) caustics. We start with a more precise characterization of the Sobolev space of maps $W^{1,2}(\widehat{\Sigma}, \Sigma)$, mentioned on passing in commenting Definition 6. To define $W^{1,2}(\widehat{\Sigma}, \Sigma)$ we follow a standard approach in harmonic map theory and use Nash embedding theorem [17,28], by considering the compact surface $\left(\Sigma_{L}, h\right)$ isometrically embedded into some Euclidean space $\mathbb{E}^{m}:=\left(\mathbb{R}^{m}, \delta\right)$ for $m$ sufficiently large. In particular, if $J:\left(\Sigma_{L}, h\right) \hookrightarrow \mathbb{E}^{m}$ is any such an embedding, then we define the Sobolev space of maps

$$
W_{(J)}^{1,2}(\widehat{\Sigma}, \Sigma):=\left\{\varphi \in W^{1,2}\left(\widehat{\Sigma}, \mathbb{R}^{m}\right) \mid \varphi\left(\hat{\Sigma}_{L}\right) \subset J\left(\Sigma_{L}\right)\right\}
$$

where $W^{1,2}\left(\widehat{\Sigma}, \mathbb{R}^{m}\right)$ is the Hilbert space of square summable $\varphi: \widehat{\Sigma} \rightarrow \mathbb{R}^{m}$, with (first) distributional derivatives in $L^{2}\left(\widehat{\Sigma}, \mathbb{R}^{m}\right)$, endowed with the norm

$$
\|\varphi\|_{W^{1,2}}:=\int_{\widehat{\Sigma}}\left(\varphi^{a}(x) \varphi^{b}(x) \delta_{a b}+\hat{h}^{\mu \nu}(x) \frac{\partial \varphi^{a}(x)}{\partial x^{\mu}} \frac{\partial \varphi^{b}(x)}{\partial x^{\nu}} \delta_{a b}\right) \mathrm{d} \mu_{\hat{h}},
$$

\footnotetext{
6 The reason for keeping $\psi_{L}$ fixed is directly related to the fact that $\psi_{L}$ it is constructed by using the null geodesics along the past lightcones by exploiting the exponential maps (see (24)), and this is the way actual cosmological observations are carried out.
} 
where, for $\varphi(x) \in J\left(\Sigma_{L}\right) \subset \mathbb{R}^{m}, a, b=1, \ldots, m$ label coordinates in $\left(\mathbb{R}^{m}, \delta\right)$, and $\mathrm{d} \mu_{\hat{h}}$ denotes the Riemannian measure on $(\widehat{\Sigma}, \hat{h})$. This characterization is independent of $J$ since $\Sigma_{L}$ is compact, and in that case for any two isometric embeddings $J_{1}$ and $J_{2}$, the corresponding spaces of maps $W_{\left(J_{1}\right)}^{1,2}(\widehat{\Sigma}, \Sigma)$ and $W_{\left(J_{2}\right)}^{1,2}(\widehat{\Sigma}, \Sigma)$ are homeomorphic [19]. For this reason, in what follows we shall simply write $W^{1,2}(\widehat{\Sigma}, \Sigma)$. The set of maps $W^{1,2}(\widehat{\Sigma}, \Sigma)$ provides the minimal regularity allowing for the characterization of the energy functional $E_{\widehat{\Sigma} \Sigma}\left[\psi_{L}, \zeta\right]$. Maps of class $W^{1,2}(\widehat{\Sigma}, \Sigma)$ are not necessarily continuous and, even if the space of smooth maps $C^{\infty}(\widehat{\Sigma}, \Sigma)$ is dense [26] in $W^{1,2}(\widehat{\Sigma}, \Sigma)$, to carry out explicit computations, in what follows we must further require that $\varphi \in W^{1,2}(\widehat{\Sigma}, \Sigma)$ is localizable (cf. [22], Sect. 8.4) and keeps track both of the given $\psi_{L}$ and of the three alignment points $\hat{q}_{(i)}, i=1,2,3$ on $\left(\widehat{\Sigma}_{L}, \hat{h}\right)$. The only freedom remaining is in the conformal group automorphisms $\zeta \in \operatorname{PSL}(2, \mathbb{C})$ acting on $\left(\widehat{\Sigma}_{L}, \hat{h}\right)$, and in terms of which we need to control that the images of the reference points $\hat{q}_{(i)} \in \widehat{\Sigma}_{L}$ stay separated and do not concentrate in a small neighborhood of $\Sigma_{L}$. Hence, and for a fixed $\psi_{L} \in W^{1,2}(\widehat{\Sigma}, \Sigma)$, we define the space of maps over which $E_{\widehat{\Sigma} \Sigma}\left[\psi_{L}, \zeta\right]$ is minimized according to the following definition.

Definition Let us assume that $\psi_{L}:\left(\widehat{\Sigma}_{L}, \hat{h}\right) \longrightarrow\left(\Sigma_{L}, h\right)$ is, for almost all points of $\widehat{\Sigma}_{L}$, a $W^{1,2}(\widehat{\Sigma}, \Sigma)$ diffeomorphism between the two celestial spheres ${ }^{7}$, and let $\hat{q}_{(i)}, i=1,2,3$, be the three distinguished points on $\left(\widehat{\Sigma}_{L}, \hat{h}\right)$, characterizing the three reference past-directed null directions on $\mathcal{C}_{L}^{-}(p, \hat{g})$ introduced in Proposition 5. A map $\varphi:=\psi_{L} \circ \zeta \in W^{1,2}(\widehat{\Sigma}, \Sigma)$, with $\zeta \in \operatorname{PSL}(2, \mathbb{C})$, is said to be $\varepsilon$-localizable if: (i) For every $\hat{q} \in \widehat{\Sigma}_{L}$ there exists a metric disks $D(\hat{q}, \delta):=\left\{y \in \widehat{\Sigma}_{L} \mid d_{\gamma}(\hat{q}, y) \leq \delta\right\} \subset \widehat{\Sigma}_{L}$, of radius $\delta>0$, with smooth boundary $\partial D$, and containing at most one of the three points $\hat{q}_{(i)}, i=1,2,3$; and (ii) corresponding to each of these disks, there exists a metric disk $B(q, \varepsilon)=\varphi(D(\hat{q}, \delta)):=\left\{x \in \Sigma_{L} \mid d_{h}(q, x) \leq \varepsilon\right\} \subset\left(\Sigma_{L}, h\right)$ centered at $\varphi\left(x_{0}\right):=q \in M$, of radius $r>0$ such that $\varphi\left(D\left(x_{0}, \delta\right)\right) \subset B(q, r)$, with $\varphi(\partial D) \subset B(q, \varepsilon)$. Under such assumptions, we consider, for fixed $\psi_{L}$, the space of maps

$$
\begin{aligned}
& \operatorname{Map}_{\psi}\left(\widehat{\Sigma}_{L}, \Sigma_{L}\right):=\left\{\varphi:=\psi_{L} \circ \zeta \in W^{1,2}(\widehat{\Sigma}, \Sigma) \cap C^{0}(\widehat{\Sigma}, \Sigma),\right. \\
& \left.\zeta \in \operatorname{PSL}(2, \mathbb{C}) \mid \varphi:=\psi_{L} \circ \zeta \text { is " - localizable and } \Phi_{\widehat{\Sigma} \Sigma}(\hat{q}) \geq 0\right\},
\end{aligned}
$$

where the non-negativity requirement $\Phi_{\widehat{\Sigma} \Sigma}(\hat{q}) \geq 0$ is assumed to hold for almost all points of $\widehat{\Sigma}_{L}$.

As in harmonic map theory, there is a further delicate issue related to the fact that maps in $\operatorname{Map}_{\psi}\left(\widehat{\Sigma}_{L}, \Sigma_{L}\right)$ are partitioned in different homotopy classes. Recall that every map from $\mathbb{S}^{2}$ into itself is characterized by the degree of the map [24], measuring how many times the map wraps $\mathbb{S}^{2}$ around itself. In particular through the action of a sequence of conformal dilations $\in \operatorname{PSL}(2, \mathbb{C})$ of the form $\zeta \longmapsto \zeta_{(k)}^{\prime}:=\omega_{(k)} \zeta$ where

\footnotetext{
7 Hence, we are assuming that there can be a finite collections of points for which the exponential mapping $\exp _{p}$ along $\mathcal{C}_{L}^{-}(p, g)$ may be not injective.
} 
$\omega_{(k)} \in \mathbb{R}$ we can easily construct sequences of mappings $\left\{\varphi_{(k)}\right\}$ that tend to focus all points of a disk $D$ in $\mathbb{S}^{2}$ toward a given point (say the north pole). Physically this corresponds to the effect of acting with a sequence of Lorentz boosts (with rapidity $\left.\log \omega_{(k)}\right)$ on an observer $P$ who is looking at the giving region $D$ of the celestial sphere. From the point of view of $P$, one can also interpret this as a focusing of the past null geodesics eventually leading to the formation of a caustic point. Regardless of the physical interpretation, in harmonic map theory this sort of behavior leads to the phenomenon of bubble convergence when discussing the minimization problem for the harmonic map energy functional [25]. In our case, we can exploit the analogous of bubbling convergence to our advantage in order to extend our analysis to the case when caustics are present. We can model the generation of caustic points as the results of a focusing mapping, such as the $\left\{\varphi_{(k)}\right\}$ described above, converging in $\operatorname{Map}_{\psi}\left(\widehat{\Sigma}_{L}, \Sigma_{L}\right)$, to a $\operatorname{deg} \varphi=h>0$ map (if there are $h-1$ caustic points in $\left(\Sigma_{L}, h\right)$ ). We postpone the details of such analysis to a paper in preparation [4], and limit here our analysis to show that $E_{\widehat{\Sigma} \Sigma}\left[\psi_{L}, \zeta\right]$ can be minimized over diffeomorphisms in $\operatorname{Map}_{\psi}\left(\widehat{\Sigma}_{L}, \Sigma_{L}\right)$.

Theorem 9 (The lightcone comparison distance at scale $L$ ). The functional $E_{\widehat{\Sigma} \Sigma}\left[\psi_{L}, \zeta\right]$ achieves a minimum on $\operatorname{Map}_{\psi}\left(\widehat{\Sigma}_{L}, \Sigma_{L}\right)$, and

$$
d_{L}\left[\widehat{\Sigma}_{L}, \Sigma_{L}\right]:=\inf _{\psi_{L} \circ \zeta \in \operatorname{Map}_{\psi}\left(\widehat{\Sigma}_{L}, \Sigma_{L}\right)} E_{\widehat{\Sigma} \Sigma}\left[\psi_{L}, \zeta\right]
$$

defines a scale-dependent distance between the celestial spheres $\left(\widehat{\Sigma}_{L}, \hat{h}\right)$ and $\left(\Sigma_{L}, h\right)$ on the lightcone regions $\mathcal{C}_{L}^{-}(p, \hat{g})$ and $\mathcal{C}_{L}^{-}(p, g)$.

Proof To simplify notation let us set $\varphi:=\psi_{L} \circ \zeta$. Since we have the upper bound $E_{\widehat{\Sigma} \Sigma}[\varphi] \leq C_{L}:=A\left(\widehat{\Sigma}_{L}\right)+A\left(\Sigma_{L}\right)$, (see (52)), we can limit our analysis to the subset of maps

$$
\operatorname{Map}_{\psi, C_{L}}\left(\widehat{\Sigma}_{L}, \Sigma_{L}\right):=\left\{\varphi \in \operatorname{Map}_{\psi}\left(\widehat{\Sigma}_{L}, \Sigma_{L}\right) \mid \text { s.t. } E_{\widehat{\Sigma} \Sigma}[\varphi] \leq C_{L}\right\} \text {. }
$$

According to Definition 4, the space of maps $\operatorname{Map}_{\varepsilon, C_{L}}(\widehat{\Sigma}, \Sigma)$ is equicontinuous, namely for any point $\hat{q} \in \hat{\Sigma}$ we can choose the disk $D\left(\hat{q}_{1}, \delta\right)$ (for notation see Definition 4) in such a way that for a given $\varepsilon>0, \varphi\left(\hat{q}_{1}\right)$ and $\varphi\left(\hat{q}_{2}\right)$ are such that $d_{h}\left(\varphi\left(\hat{q}_{1}\right), \varphi\left(\hat{q}_{2}\right)\right)<\varepsilon$, for all $\hat{q}_{2} \in D\left(\hat{q}_{1}, \delta\right)$ and all $\varphi \in \operatorname{Map}_{\psi, C_{L}}\left(\widehat{\Sigma}_{L}, \Sigma_{L}\right)$. Hence, a minimizing sequence $\left\{\varphi_{(k)}\right\}_{k \in \mathbb{N}} \in \operatorname{Map}_{\varepsilon, C_{L}}(\widehat{\Sigma}, \Sigma)$ for $E_{\widehat{\Sigma} \Sigma}[\varphi]$ is equicontinuous. By selecting a subsequence we may assume that $\left\{\varphi_{(k)}\right\}$ converges to a continuous map $\varphi$ which is also the weak limit of $\left\{\varphi_{(k)}\right\}$ in $W^{1,2}(\widehat{\Sigma}, \Sigma)$, since this latter is a weakly compact space of maps.

Since

$$
E_{\widehat{\Sigma} \Sigma}[\varphi]=A\left(\Sigma_{L}\right)+A\left(\widehat{\Sigma}_{L}\right)-2 \int_{\widehat{\Sigma}_{L}} \Phi_{\widehat{\Sigma} \Sigma}(\varphi) \mathrm{d} \mu_{\hat{h}},
$$

a minimizing (sub)sequence $\left\{\varphi_{(k)}\right\}$ for $E_{\widehat{\Sigma} \Sigma}[\varphi]$ corresponds to a maximize sequence for the functional $\int_{\widehat{\Sigma}_{L}} \Phi_{\widehat{\Sigma} \Sigma}(\varphi) \mathrm{d} \mu_{\hat{h}}$. Hence, given $\delta>0$, there exists $k_{0}$ such that for 
all $k \geq k_{0}$, we have

$$
\int_{\widehat{\Sigma}_{L}} \Phi_{\widehat{\Sigma} \Sigma}(\bar{\varphi}) \mathrm{d} \mu_{\hat{h}} \geq \int_{\widehat{\Sigma}_{L}} \Phi_{\widehat{\Sigma} \Sigma}\left(\varphi_{(k)}\right) \mathrm{d} \mu_{\hat{h}}-\delta
$$

along a minimizing sequence $\left\{\varphi_{(k)}\right\} \longrightarrow \bar{\varphi}$ for the functional $E_{\widehat{\Sigma} \Sigma}[\varphi]$, and where $\Phi_{\widehat{\Sigma} \Sigma}\left(\varphi_{(k)}\right)$ is non-negative for almost all points of $\widehat{\Sigma}_{L}$. By adding and subtracting $\int_{\widehat{\Sigma}_{L}} \Phi_{\widehat{\Sigma} \Sigma}\left(\varphi_{(k)}\right) \mathrm{d} \mu_{\hat{h}}$ to (77), (evaluated for $\bar{\varphi}$ ), and by taking into account (78), we get

$$
E_{\widehat{\Sigma} \Sigma}[\bar{\varphi}]=E_{\widehat{\Sigma} \Sigma}\left[\varphi_{(k)}\right]-2 \int_{\widehat{\Sigma}_{L}}\left(\Phi_{\widehat{\Sigma} \Sigma}(\bar{\varphi})-\Phi_{\widehat{\Sigma} \Sigma}\left(\varphi_{(k)}\right)\right) \mathrm{d} \mu_{\hat{h}} \leq E_{\widehat{\Sigma} \Sigma}\left[\varphi_{(k)}\right]+2 \delta
$$

for all $k \geq k_{0}$. Since the choice of $\delta>0$ is arbitrary, (79) implies that the functional $E_{\widehat{\Sigma} \Sigma}[\varphi]$ is lower semicontinuous, i.e.,

$$
E_{\widehat{\Sigma} \Sigma}[\bar{\varphi}] \leq \lim _{k} \inf E_{\widehat{\Sigma} \Sigma}\left[\varphi_{k}\right]
$$

for all $\varphi \in \operatorname{Map}_{\varepsilon, C_{L}}(\widehat{\Sigma}, \Sigma)$ with $\varphi_{k}$ weakly converging, in the above sense, to $\bar{\varphi}$. Hence, $\left\{\varphi_{(k)}\right\} \longrightarrow \bar{\varphi}$ minimizes $E_{\widehat{\Sigma} \Sigma}[\varphi]$ in the space of maps $\operatorname{Map}_{\varepsilon}(\widehat{\Sigma}, \Sigma)$, as stated.

If we set

$$
d_{L}\left[\widehat{\Sigma}_{L}, \Sigma_{L}\right]:=\inf _{\psi_{L} \circ \zeta \in \operatorname{Map}_{\varepsilon}(\widehat{\Sigma}, \Sigma)} E_{\widehat{\Sigma} \Sigma}\left[\psi_{L}, \zeta\right]
$$

then as a consequence of the properties of the functional $E_{\widehat{\Sigma} \Sigma}[\varphi]$, described in Lemma 8 , we have that $d_{L}\left[\widehat{\Sigma}_{L}, \Sigma_{L}\right]$ provides a scale-dependent distance function between the physical celestial sphere $\left(\Sigma_{L}, h\right)$ and the reference FLRW celestial sphere $\left(\widehat{\Sigma}_{L}, \hat{h}\right)$, as the scale $L$ varies. In particular, with the notation of Lemma 8 we have (i)Nonnegativity $d_{L}\left[\widehat{\Sigma}_{L}, \Sigma_{L}\right] \geq 0$; (ii) $d_{L}\left[\widehat{\Sigma}_{L}, \Sigma_{L}\right]=0$ iff $\left(\widehat{\Sigma}_{L}, \hat{h}\right)$ and $\left(\Sigma_{L}, h\right)$ are isometric; (iii) Symmetry $d_{L}\left[\widehat{\Sigma}_{L}, \Sigma_{L}\right]=d_{L}\left[\Sigma_{L}, \widehat{\Sigma}_{L}\right]$; (iv) Triangular inequality $d_{L}\left[\widehat{\Sigma}_{L}, \widetilde{\Sigma}_{L}\right] \leq d_{L}\left[\hat{\Sigma}_{L}, \Sigma_{L}\right]+d_{L}\left[\Sigma_{L}, \widetilde{\Sigma}_{L}\right]$.

\section{Causal diamonds and the physical meaning of $d_{L}\left[\widehat{\Sigma}_{L}, \Sigma_{L}\right]$}

The distance functional $d_{L}\left[\widehat{\Sigma}_{L}, \Sigma_{L}\right]$ is a geometric quantity that we can associate with the observer who wishes to describe with a Friedmannian bias the cosmological region where inhomogeneities may dominate. To appreciate what this role implies, let us briefly discuss the physical interpretation of $d_{L}\left[\widehat{\Sigma}_{L}, \Sigma_{L}\right]$, when we probe the light cone regions $\mathcal{C}_{L}^{-}(p, \hat{g})$ and $\mathcal{C}_{L}^{-}(p, g)$ over a sufficiently small length scale $L$. If $\bar{\varphi}$ denotes the minimizing map characterized in Theorem 9, we can write

$$
d_{L}\left[\widehat{\Sigma}_{L}, \Sigma_{L}\right]=E_{\widehat{\Sigma} \Sigma}[\bar{\varphi}]:=A\left(\widehat{\Sigma}_{L}\right)+A\left(\Sigma_{L}\right)-2 \int_{\widehat{\Sigma}_{L}} \Phi_{\widehat{\Sigma} \Sigma} \mathrm{d} \mu_{\hat{h}}
$$


To simplify matters, we assume that at the given length scale $L$ the corresponding region $\mathcal{C}_{L}^{-}(p, g)$ is caustic-free, and parametrize $\Phi_{\Sigma \widehat{\Sigma}}(\bar{\varphi})$ as

$$
\Phi_{\Sigma \widehat{\Sigma}}(\bar{\varphi})=1+F(\bar{\varphi})
$$

where $F(\bar{\varphi})$ is a smooth function (not necessarily positive) which, by discarding the traceless lensing shear, may be thought of as describing the (small) local isotropic focusing distortion of the images of the astrophysical sources on $(\Sigma, h)$ due to gravitational lensing (see the expression (12) of the sky-mapping metric $h$ ). Under these assumptions, we can write (82) as

$$
d_{L}\left[\widehat{\Sigma}_{L}, \Sigma_{L}\right]=A\left(\Sigma_{L}\right)-A\left(\widehat{\Sigma}_{L}\right)-2 \int_{\widehat{\Sigma}_{L}} F(\bar{\varphi}) \mathrm{d} \mu_{\hat{h}}
$$

This expression can be further specialized if we exploit the asymptotic expressions of the area $A\left(\widehat{\Sigma}_{L}\right)$ and $A\left(\Sigma_{L}\right)$ of the two surfaces $\left(\widehat{\Sigma}_{L}, \hat{h}\right),\left(\Sigma_{L}, h\right)$ on the corresponding lightcones $\mathcal{C}_{L}^{-}(p, \hat{g})$ and $\mathcal{C}_{L}^{-}(p, g)$. These asymptotic expressions can be obtained if we consider the associated causal past regions $\mathcal{J}_{L}^{-}(p, \hat{g})$ and $\mathcal{J}_{L}^{-}(p, g)$ sufficiently near the (common) observation point $p$, in particular when the length scale $L$ we are probing is small with respect to the "cosmological" curvature scale. Under such assumption, there is a unique maximal 3-dimensional region $V_{L}^{3}(p)$, embedded in $\mathcal{J}_{L}^{-}(p, g)$, having the surface $\left(\Sigma_{L}, h\right)$ as its boundary. This surface intersects the worldline $\gamma(\tau)$ of the observer $p$ at the point $q=\gamma\left(\tau_{0}=-L\right)$ defined by the given length scale $L$. For the reference FLRW the analogous setup is associated with the constant-time slicing of the FLRW spacetime $(M, \hat{g})$ considered. The corresponding three-dimensional region $\widehat{V}_{L}^{3}(p)$, embedded in $\mathcal{J}_{L}^{-}(p, \hat{g})$, has the surface $\left(\widehat{\Sigma}_{L}, \hat{h}\right)$ as its boundary. The FLRW observer $\hat{\gamma}(\hat{\tau})$ will intersect $\widehat{V}_{L}^{3}(p)$ at the point $\hat{q}=\hat{\gamma}\left(\hat{\tau}_{0}=-L\right)$. By introducing geodesic normal coordinates $\left\{X^{i}\right\}$ in $\mathcal{J}_{L}^{-}(p, g)$ and $\left\{Y^{k}\right\}$ in $\mathcal{J}_{L}^{-}(p, \hat{g})$, respectively, based at the point $q$ and $\hat{q}$, we can pull back the metric tensors $g$ and $\hat{g}$ to $T_{q} M$ and $T_{\hat{q}} M$, and obtain the classical normal coordinate development of the metrics $g$ and $\hat{g}$ valid in a sufficiently small convex neighborhood of $q$ and $\hat{q}$. Explicitly, for the (more relevant case of the) metric $g$, we have (see, e. g., Lemma 3.4 (p. 210) of [27] or [24])

$$
\begin{gathered}
\left(\left(\exp _{q}\right)^{*} g\right)_{e f}=\eta_{e f}-\left.\frac{1}{3} \mathrm{R}_{e a b f}\right|_{q} X^{a} X^{b}-\left.\frac{1}{6} \nabla_{c} \mathrm{R}_{e a b f}\right|_{q} X^{a} X^{b} X^{c} \\
+\left(-\frac{1}{20} \nabla_{c} \nabla_{\mathrm{d}} \mathrm{R}_{e a b f}+\frac{2}{45} \mathrm{R}_{e a b m} \mathrm{R}_{f c d}^{m}\right)_{q} X^{a} X^{b} X^{c} X^{\mathrm{d}}+\ldots
\end{gathered}
$$

where $\mathrm{R}_{a b c d}$ is the Riemann tensor of the metric $g$ (evaluated at the point $q$ ). The induced expansion in the pulled-back measure $\left(\left(\exp _{s(\eta)}\right) * \mathrm{~d} \mu_{g}\right)$ provides the Lorentzian analog of the familiar Bertrand-Puiseux formulas associated with the geometrical interpretation of the sectional, Ricci and scalar curvature for a Riemannian manifold in terms of the length, area, and volume measures of small geodesic balls. In the Lorentzian case the relevant formulas are more delicate to derive, and their analysis 
has given rise to a deep line of research where the role of Riemannian geodesic balls is taken over by the spacetime geometry of small causal diamonds. The study of these Lorentzian avatars of Riemannian balls was presciently initiated by Myrheim [23], and their properties are finely described in [13] (see also [2,14]). That said, small causal diamonds asymptotics [13] provides, to leading order in $L$, the following expressions for the area of $\left(\Sigma_{L}, h\right)$ and $\left(\widehat{\Sigma}_{L}, \hat{h}\right)$,

$$
A\left(\Sigma_{L}\right)=\pi L^{2}\left(1-\frac{1}{72} L^{2} \mathrm{R}(q)+\ldots\right),
$$

and

$$
A\left(\widehat{\Sigma}_{L}\right)=\pi L^{2}\left(1-\frac{1}{72} L^{2} \widehat{\mathrm{R}}(\hat{q})+\ldots\right),
$$

Introducing these expressions in (84), we get

$$
d_{L}\left[\widehat{\Sigma}_{L}, \Sigma_{L}\right]=\frac{\pi}{72} L^{4}(\widehat{\mathrm{R}}(\hat{q})-\mathrm{R}(q))-2 \int_{\widehat{\Sigma}_{L}} F(\bar{\varphi}) \mathrm{d} \mu_{\hat{h}}+\ldots
$$

We can rewrite this equivalently as

$$
\widehat{\mathrm{R}}(\hat{q})=\mathrm{R}(q)+\frac{72}{\pi} \frac{d_{L}\left[\widehat{\Sigma}_{L}, \Sigma_{L}\right]}{L^{4}}+\frac{144}{\pi L^{4}} \int_{\widehat{\Sigma}_{L}} F(\bar{\varphi}) \mathrm{d} \mu_{\hat{h}}+\ldots
$$

The asymptotics (87) (but also the very characterization of $d_{L}\left[\widehat{\Sigma}_{L}, \Sigma_{L}\right]$ ), shows clearly that the lightcone comparison functional $E_{\widehat{\Sigma} \Sigma}$ and the associated distance $d_{L}\left[\widehat{\Sigma}_{L}, \Sigma_{L}\right]$ provide a generalization of the lightcone theorem [7] proved by $\mathrm{Y}$. Choquet-Bruhat, P. T. Chrusciel, and J. M. Martin-Garcia. As suggested in Introduction, the normal coordinates asymptotics (88) explicitly relating $d_{L}\left[\widehat{\Sigma}_{L}, \Sigma_{L}\right]$, the average lensing shear and the scalar curvatures $\widehat{\mathrm{R}}(\hat{q})$ and $\mathrm{R}(q)$, is a strong indication of the existence of a deeper relation between the geometric analysis approach to lightcones comparison discussed here and the comparison theorems for causal diamonds discussed by Berthiere et al. [2]. Motivations and techniques characterizing the two approaches are clearly very different, but the analysis of Bishop's inequality in [2] is strongly indicative of the possibility of introducing a lightcone distance functional also within the causal diamond formalism. In Riemannian geometry, Bishop's inequality is indeed instrumental to the characterization of the Gromov-Hausdorff distance between Riemannian manifolds [15], and currently there are optimal transport approaches to establishing a Lorentzian Bishop-Gromov inequality (see, e.g., [6]). In this connection is perhaps worthwhile to stress that the variational characterization of our distance functional $d_{L}\left[\widehat{\Sigma}_{L}, \Sigma_{L}\right]$, which remains valid also for lightcone sections with caustics, complies with optimal transport techniques since the harmonic type functional $E_{\widehat{\Sigma} \Sigma}$ can be rephrased within such a formalism (see, e.g., [5] for an optimal transport transcription of a functional similar to $E_{\widehat{\Sigma}} \Sigma$ ). This varied landscape is indicative of a potential common ground between our approach and that causal diamond theory. This remains, however, as an open problem worthwhile of further investigation. 
Further interest in the normal coordinates asymptotics (88) lies in the fact that it directly connects $d_{L}\left[\widehat{\Sigma}_{L}, \Sigma_{L}\right]$ to the fluctuations in the spacetime scalar curvature: if we decide to keep on in modeling with a FLRW solution a cosmological spacetime, homogeneous on large scale but highly inhomogeneous at smaller scale, then the associated scalar curvature $\widehat{\mathrm{R}}(\hat{q})$ can be approximately identified with the physical scalar curvature $\mathrm{R}(\hat{q})$, with a rigorous level of scale dependence precision, only if we take into account the contribution provided by the lightcone distance functional $d_{L}\left[\widehat{\Sigma}_{L}, \Sigma_{L}\right]$ (and by the average of the local focusing term $F(\bar{\varphi})$ ). This can be of relevance in addressing backreaction problems in cosmology (see, e.g., [3]). Finally, $d_{L}\left[\widehat{\Sigma}_{L}, \Sigma_{L}\right]$ can be also of some use in providing a rigorous way of addressing some aspect of the best-fitting problem in cosmology (see $[10,11]$ ), roughly speaking, the strategy is to vary the family of model spacetimes $(M, \hat{g})$ (for instance, the family of FLRW solutions, or the larger family of homogeneous spacetimes) in such a way to minimize (over the relevant interval of length scales $L$ ) the distance functional $d_{L}\left[\widehat{\Sigma}_{L}, \Sigma_{L}\right]$ between the physical celestial spheres $\left(\widehat{\Sigma}_{L}, \hat{h}\right)$ and the family of reference celestial spheres $\left(\widehat{\Sigma}_{L}, \hat{h}\right)$ associated with the model spacetimes $(M, \hat{g})$ adopted.

Acknowledgements We wish to thank Thomas Buchert and Dennis Stock for valuable discussions. We would also like to thank the reviewers for their thoughtful comments. This work has been partially supported by the European project ERC advanced grant 740021 "ARThUs".

Funding Information Open access funding provided by Università degli Studi di Pavia within the CRUICARE Agreement.

Open Access This article is licensed under a Creative Commons Attribution 4.0 International License, which permits use, sharing, adaptation, distribution and reproduction in any medium or format, as long as you give appropriate credit to the original author(s) and the source, provide a link to the Creative Commons licence, and indicate if changes were made. The images or other third party material in this article are included in the article's Creative Commons licence, unless indicated otherwise in a credit line to the material. If material is not included in the article's Creative Commons licence and your intended use is not permitted by statutory regulation or exceeds the permitted use, you will need to obtain permission directly from the copyright holder. To view a copy of this licence, visit http://creativecommons.org/licenses/by/4.0/.

\section{References}

1. Berger, M.S.: On Riemannian structures of prescribed Gaussian curvature for compact 2-manifolds. J. Diff. Geo. 5, 325-332 (1971)

2. Berthiere, C., Gibbons, G., Solodukhin, S.N.: Comparison theorems for causal diamonds. Phys. Rev. D 92, 064036 (2015)

3. Buchert, T., Carfora, M., Ellis, G.F.R., Kolb, E.W., MacCallum, M., Ostrowski, J., Rasanen, S., Roukema, B., Andersson, L., Coley, A., Wiltshire, D.: Is there proof that backreaction of inhomogeneities is irrelevant in cosmology? Class. Quant. Gravity 32(21), 215021 (2015)

4. Carfora, M., Familiari, F.: A comparison theorem for cosmological lightcones. Caustics and weak lensing, in preparation

5. Carfora, M.: The Wasserstein geometry of nonlinear sigma models and the Hamilton-Perelman Ricci flow. Rev. Math. Phys. 29(1), 1750001 (2017). https://doi.org/10.1142/S0129055X17500015

6. Cavalletti, F., Mondino, A.: Optimal transport in Lorentzian synthetic spaces, synthetic timelike Ricci curvature lower bounds and applications. arXiv:2004.08934v2 [math.MG] 3 Dec (2020)

7. Choquet-Bruhat, Y., Chrusciel, P.T., Martin-Garcia, J.M.: The light-cone theorem. Class. Quantum Grav. 26, 135011 (2009) 
8. Choquet-Bruhat, Y., Chrusciel, P.T., Martin-Garcia, J.M.: The Cauchy problem on a characteristic cone for the Einstein equations in arbitrary dimensions. Ann Henri Poincare 12, 419-482 (2011)

9. Dubrovin, B. A., Fomenko, A. T., Novikov, S.P.: Modern Geometry-Methods and Applications: Part I: The Geometry of Surfaces, Transformation Groups, and Fields (1984, 1992); Part II: The Geometry and Topology of Manifolds (1985); Part III: Introduction to Homology Theory. Springer, Berlin (1990)

10. Ellis, G.F.R., Nel, S.D., Maartens, R., Stoeger, W.R., Whitman, A.P.: Ideal observational cosmology. Phys. Rep. 124, 315-417 (1985)

11. Ellis, G.F.R., Maartens, R., MacCallum, M.A.H.: Relativistic Cosmology. Cambridge University Press, Cambrige (2012)

12. Fleury, P., Nugier, F., Fanizza, G.: Geodesic-light-cone coordinates and the Bianchi I spacetime. J. Cosmol. Astroparticle Phys. (JCAP) 06, 008 (2016)

13. Gibbons, G.W., Solodukhin, S.N.: The geometry of small causal diamonds. Phys. Lett. B 649, 317 (2007). [hep-th/0703098]

14. Gibbons, G.W., Solodukhin, S.N.: The geometry of large causal diamonds and the No-Hair property of asymptotically DeSitter spacetimes. Phys. Lett. B 652, 103 (2007). [arXiv: 0706.0603]

15. Gromov, M.: Metric Structures for Riemannian and Non-Riemannian Spaces, Progress in Mathematics. Birkhäuser, Boston (1999)

16. Gu, X., Yau, S.-T.: Computing conformal structure of surfaces. Commun. Inf. Syst. 2, 121-146 (2002)

17. Günther, M.: On the perturbation problem associated to isometric embeddings of Riemannian manifolds. Ann. Global Anal. Geom. 7, 69-77 (1989)

18. Hass, J., Koehl, P.: Comparing shapes of genus-zero surfaces. J. Appl. Comput. Topol. 1, 57-87 (2017)

19. Hélein, F., Wood, J.C.: Harmonic Maps, in Handbook of Global Analysis. Elsevier, Amsterdam (2007)

20. Jin, M., Wang, Y., Yau, S.-T., Gu, X.: Optimal global conformal surface parametrization for visualization. Commun. Inf. Syst. 117-134 (2005)

21. Kolb, E.W., Marra, V., Matarrese, S.: Cosmological background solutions and cosmological backreactions. Gen. Rel. Grav. 42, 1399-1412 (2010)

22. Jost, J.: Riemannian Geometry and Geometric Analysis, 2nd edn. Springer Universitext, SpringerVerlag, Berlin (1998)

23. Myrheim, J.: Statistical Geometry, Report No. CERN-TH-2538, (1978) (unpublished)

24. Petersen, P.: Riemannian Geometry, Graduate Text in Mathematics 171 Springer (1998)

25. Sacks, J., Uhlenbeck, K.: The existence of minimal immersions of 2-spheres. Ann. Math. 113, 1-24 (1981)

26. Schoen, R., Uhlenbeck, K.: The Dirichlet problem for harmonic maps. J. Differ. Geom. 18, 253-268 (1983)

27. Schoen, R., Yau, S.-T.: Lectures on Differential Geometry, vol. I. International Press, Cambridge, MA (1994)

28. Schwartz, J.T.: Non Linear Functional Analysis. Gordon and Breach, New York (1969)

Publisher's Note Springer Nature remains neutral with regard to jurisdictional claims in published maps and institutional affiliations. 